\title{
Flooding and Drying in Discontinuous Galerkin Finite-Element Discretizations of Shallow-Water Equations. Part 1: One Dimension.
}

\author{
Onno Bokhove \\ Department of Applied Mathematics \\ 7500 AE, P.O. Box 217, Enschede \\ University of Twente, The Netherlands \\ o.bokhove@math.utwente.nl
}

January 14, 2004

\begin{abstract}
Free boundaries in shallow-water equations demarcate the time-dependent water line between "flooded" and "dry" regions. We present a novel numerical algorithm to treat flooding and drying in a formally second-order explicit space discontinuous Galerkin finite-element discretization of the one-dimensional or symmetric shallow-water equations. The algorithm uses fixed Eulerian flooded elements and a mixed Eulerian-Lagrangian element at each free boundary. When the time step is suitably restricted, we show that the mean water depth is positive. This time step restriction is based on an analysis of the discretized continuity equation while using the HLLC flux. The algorithm and its implementation are tested in comparison with a large and relevant suite of known exact solutions. The essence of the flooding and drying algorithm pivots around the analysis of a continuity equation with a fluid velocity and a pseudo density (in the shallow water case the depth). It therefore also applies, for example, to space discontinuous Galerkin finite-element discretizations of the compressible Euler equations in which vacuum regions emerge, in analogy of the above dry regions. We believe that the approach presented can be extended to finitevolume discretizations with similar mean level and slope reconstruction.
\end{abstract}

Keywords: shallow-water equations, flooding and drying, free-boundary dynamics, discontinuous Galerkin finite-element method, positivity of mean water depth

Running head: Flooding and drying in DGFEM

AMS Subject Classification: 76M10, 65M60

Under consideration for publication in J. Sci. Comp. 


\section{Introduction}

We consider explicit space discontinuous Galerkin finite-element discretizations of the symmetric or one-dimensional shallow-water equations (e.g., Pedlosky, 1987)

$$
\begin{aligned}
\partial_{t} h+\partial_{x}(h u) & =0 \\
\partial_{t}(h u)+\partial_{x}\left(h u^{2}+P\right) & =S_{2} \equiv-g h \partial_{x} h_{b}+S_{2}^{*} \\
\partial_{t}(h v)+\partial_{x}(h u v) & =S_{3}
\end{aligned}
$$

with the horizontal velocity field $(u, v)=(u(x, t), v(x, t))$ as function of the horizontal coordinate $x$ and time $t ; h(x, t)$ the depth of the layer of water; $P=P(h)=g h^{2} / 2$ the "pressure" or potential; and $g$ the gravitational acceleration. The terms $S_{2}, S_{3}$ may depend in general on $x$ and $t$ explicitly, and on the variables $u, v, h$. The fluid's bottom is at $z=h_{b}(x)$. Partial derivatives are denoted by $\partial_{t}=\partial / \partial t$, etc.

This system, which is hyperbolic in its conservative limit, models the flow of a layer of water whose depth is small relative to the horizontal scales of interest. The flow domain is defined by the area where the depth $h$ is positive. In many practical applications, the flow domain may have "free" boundaries, where $h \downarrow 0$, as well as more conventional fixed boundaries (e.g., impenetrable walls). The location of this free boundary is time-dependent due to the motion of the fluid, resulting in "flooding" and "drying". Accurate prediction of this flooding and drying is important in forecasting river hydraulics, tsunamis, and near-shore surf zone dynamics on beaches and sand banks, as well as in designing dikes. This paper presents the design and verification of a discontinuous Galerkin finite-element approximation to model such flows in one space dimension, with an emphasis on the treatment of flooding and drying. ${ }^{1}$

Discontinuous Galerkin finite-element schemes have several advantages (e.g., Cockburn et al., 1989; Cockburn and Shu, 1998):

i. The structure of the scheme allows varying orders of accuracy in the elements (so-called $p$-adaptivity).

ii. It is straightforward to use elements with local mesh refinement (so-called $h$ adaptivity).

iii. The scheme is extremely local since communication occurs entirely through fluxes at the faces between elements. This property is used to deal efficiently with the free-boundary dynamics in this paper; in addition, it allows for efficient parallelization. The implementation of other boundary conditions such as inand outflow conditions is also efficient and accurate due to the local nature of the scheme.

Disadvantages are that the scheme is more complex, and that more degrees of freedom are involved relative to finite-volume or finite-difference schemes.

The flooding and drying algorithm consists of the following. To deal with discontinuities such as hydraulic jumps and bores, the depth and velocity times depth

\footnotetext{
${ }^{1} \mathrm{~A}$ preliminary version was presented in Bokhove and Wirosoetisno (2003).
} 
are the computational variables advanced in time. Nevertheless, velocity and depth are approximated by the same order polynomial basis functions per element. Consequently, the velocity times depth has a constrained, higher-order basis function. This ensures that it becomes zero at the water line exactly when the depth becomes zero, conforming to the mathematical shallow-water model and reality. The finite-element discretization is therefore Galerkin for the variables velocity and height, but not in the primary computational variables. The one-dimensional computational domain may contain multiple disconnected "patches" of water, the flooded regions, separated by dry regions. For each patch the boundary consists either of the computational boundary or a free boundary. The algorithm uses fixed Eulerian flooded elements and one mixed Eulerian-Lagrangian element at each free boundary. Hence, it is a particular case of an arbitrary Lagrangian Eulerian or ALE grid, e.g., Van der Vegt and Van der Ven (2002). Positivity of the mean water depth is ensured by a suitable restriction of the time step. This restriction is based on analysis of the discretized continuity equation in which we use the numerical HLLC flux. The size of the freeboundary element is controlled by elements which merge and split using the mean and slope information of the velocity and depth. Drying in a patch occurs when a dry region emerges in a flooded region resulting in the splitting of the original patch. Merging of patches arises when a dry region between two patches becomes inundated. Drying and merging of multiple patches of fluid is introduced using mean and slope information.

The time-step restriction we have found, is similar to that of Perthame (1999) which restriction was derived for a first-order finite-volume method with a forward Euler time discretization and a flux based on kinetic theory for the compressible Euler equations. We have adapted the kinetic flux to shallow-water equations and to our flooding and drying treatment in a discontinuous Galerkin finite-element method higher-order in space and time, but preliminary tests showed the HLLC flux to be more accurate. Perthame and Simeoni (2001) and Audusse and Bristeau (2003) use a kinetic scheme for the shallow-water equations in a finite-volume method. In particular, their algorithm preserves flow at rest over topography and provides an energy dissipation inequality. Our scheme by construction preserves flow at rest above arbitrary (piece-wise linear and smooth) topography. But so far we are unable to prove an energy dissipation inequality for our algorithm.

We verified the numerical discretization of the shallow-water equations by comparing the numerical solutions with various one-dimensional exact solutions in which flooding and drying occurs: Riemann problems with drying (e.g., Smoller, 1994, and Toro, 1999), the Carrier-Greenspan (1958) solution, the parabolic bowl solution (Young, 1986), and the Peregrine-Williams (2001) solution. This suite of exact verification cases is much more extensive than usual for testing shallow-water discretizations. The design of the algorithm has benefitted from the various test criteria.

Extra numerical dissipation may be required to control unwanted oscillations at discontinuities such as hydraulic jumps and bores. The inclusion of a dissipation operator, analogous to the one in Jaffre et al. (1995) and Van der Vegt and Van der Ven (2002) for the space-time discontinuous Galerkin finite-element discretization, is 
preferable to a slope limiter for steady state solutions. Unfortunately, we were unable to derive a dissipation operator adequate enough for the variety of complex simulations we considered. In the end, we successfully used the slope limiting treatment of Schwanenberg (2003) for the shallow-water equations with topography, and the general shock detection and limiting approach of Krivodonova et al. (2003). Since a rigorous mathematical justification of such extra numerical dissipation appears to be lacking, the addition of extra dissipation is only discussed in Appendix B as it is not required to test the flooding and drying algorithm. In contrast, the kinetic flux used by Perthame (1999) or shallow-water modifications thereof appears to have the advantage that no additional slope limiter or extra dissipation is required. Here, slope limiting is only used in one simulation of waves overtopping a dike in $\S 7$.

The paper is organized as follows. The shallow-water equations are rewritten in $\S 2$. The space discontinuous Galerkin finite-element method is set up for elements with fixed and moving (free-boundary) nodes in $\S 3$. The flux on these nodes is approximated by the HLLC flux, as explained in $\S 4$. The discretization in the special elements next to free boundaries is introduced in $\S 5$. When used with a time-step restriction, the mean water depth can be guaranteed to remain non-negative. We have thus developed a robust and accurate method that deals simultaneously with flooding and drying, and discontinuities such as hydraulic bores and jumps. In $\S 6$, we present the comparison of numerical and known exact solutions. Finally, the potential of the discretization is illustrated in a complex simulation of waves which are forced on one (offshore) boundary, steepen into bores, and subsequently flow over a dike periodically in $\S 7$. We conclude with a discussion in $\S 8$.

\section{Shallow-Water Equations}

Let $U=\left(h, m_{u}=h u, m_{v}=h v\right)^{\mathrm{T}}$. To facilitate the numerical formulation, we rewrite (1.1) concisely as

$$
\partial_{t} U+\partial_{x} F(U)=S,
$$

where $F(U)=\left(h u, h u^{2}+P, h u v\right)^{\mathrm{T}}$ is the flux, and $S=\left(0, S_{2}, S_{3}\right)^{\mathrm{T}}$ the additional topographic, dissipative or forcing term.

The flow domain $\Omega_{f} \subseteq \Omega \subset \mathbb{R}$, which may be time dependent, is embedded in a fixed computational domain $\Omega$. The boundary $\partial \Omega_{f}$ (isolated points in our onedimensional case) consists in general of fixed and free points. At the fixed boundary points, the boundary conditions specify the in- and outflow, or no through flow at walls. The free boundary is specified by the position(s) $x$ for which $h(x, t) \downarrow 0$. The system (1.1) or (2.1) is completed with initial conditions $U_{0}=U(x, 0)$. 


\section{Discontinuous Galerkin Finite-Element Method}

\subsection{Finite elements}

The flow domain $\Omega_{f} \subset \Omega=[a, b]$ is partitioned by points $x_{k}(t), k=1, \cdots, N_{\mathrm{el}}+1$, into $N_{\text {el }}$ open elements $K_{k}=\left(x_{k}, x_{k+1}\right)$. The result is a tessellation

$$
\mathcal{T}_{h}=\left\{K_{k} \mid \bigcup_{k=1}^{N_{\mathrm{el}}} \bar{K}_{k}=\bar{\Omega}_{f} \text { and } K_{k} \cap K_{k^{\prime}}=\emptyset \text { if } k \neq k^{\prime}, 1 \leq k, k^{\prime} \leq N_{\mathrm{el}}\right\}
$$

with $\bar{K}_{k}$ the closure of $K_{k}$. For convenience, we will also use the notation $x_{k, L}:=x_{k}$ and $x_{k, R}:=x_{k+1}$ below; we define $\left|K_{k}\right|=x_{k, R}-x_{k, L}$. Both $K_{k}$ and $N_{\text {el }}$ may be time-dependent, and the domain $\Omega_{f}$ may consist of disjoint "patches".

We consider finite-element discretizations of (2.1) with approximations $U_{h}$ and $w_{h}$ to the state vector $U$ and test functions $w$. These are such that $U_{h}$ and $w_{h}$ belong to the broken space

$$
V_{h}=\left\{w:\left.w\right|_{K_{k}} \in P^{d_{P}}\left(K_{k}\right), k=1, \ldots, N_{\mathrm{el}}\right\},
$$

in which $P^{d_{P}}\left(K_{k}\right)$ denotes the space of polynomials in $K_{k}$ of degree $d_{P}=d_{P}(U)$. Hence, in each element $K_{k}$, we approximate $U$ and $w$ by a polynomial spanned by basis functions $\left\{\psi_{n, k}(x, t)\right\}$ defined in $\S 3.3$ below. The complications arising from the free boundaries are discussed later, in $\S 5$.

\subsection{Weak formulation}

When we multiply (2.1) by an appropriate test function $w_{h}=w_{h}(x, t)$ and integrate by parts, the following weak formulation is obtained: Find a $U_{h} \in V_{h}$ such that $\forall w_{h} \in V_{h}$ the following relation is satisfied

$$
\begin{aligned}
\sum_{k=1}^{N}\{ & \int_{K_{k}} w_{h} \partial_{t} U_{h} \mathrm{~d} x+\left[F\left(x_{k, R}^{-}\right) w_{h}\left(x_{k, R}^{-}\right)-F\left(x_{k, L}^{+}\right) w_{h}\left(x_{k, L}^{+}\right)\right] \\
& \left.-\int_{K_{k}} F \partial_{x} w_{h} \mathrm{~d} x-\int_{K_{k}} S w_{h} \mathrm{~d} x\right\}=0
\end{aligned}
$$

where $w_{h}\left(x_{k, R}^{-}\right)=\lim _{x \uparrow x_{k, R}} w_{h}(x, t)$ and $w_{h}\left(x_{k, L}^{+}\right)=\lim _{x \downarrow x_{k, L}} w_{h}(x, t)$. (Note that we only denote dependencies explicitly when confusion may arise.)

Consider a given point $x_{k+1}=x_{k, R}=x_{k+1, L}$. Since the elements $K_{k}$ are isolated from one another at this stage, $U_{l}:=U\left(x_{k, R}^{-}\right) \neq U\left(x_{k+1, L}^{+}\right)=: U_{r}$ and consequently, the flux $F\left(x_{k, R}^{-}\right) \neq F\left(x_{k+1, L}^{+}\right)$in general. This is where the numerical flux, which is at the heart of the method, comes into play. We replace $F\left(U_{l}\right)$ and $F\left(U_{r}\right)$ by a numerical flux $\tilde{F}\left(U_{l}, U_{r}\right)$ which is consistent in the sense that $\tilde{F}(U, U)=F(U)$. Several choices are possible for $\tilde{F}$. In this paper we use the HLLC flux which is discussed in detail in section 4 . 


\subsection{Discretized weak formulation}

Within each element $K_{k}, h(x, t), u(x, t)$ and $v(x, t)$ are approximated by a polynomial, e.g.,

$$
h_{h}(x, t)=\sum_{m=0}^{d_{P}} \hat{h}_{m, k}(t) \psi_{m, k}(x, t)
$$

and similarly for the test function $w$. In a reference element $\hat{K}$ the coordinate $\zeta \in$ $(-1,1)$ is introduced with $x=x(\zeta)=\left(x_{k+1}+x_{k}\right) / 2+\left|K_{k}\right| \zeta / 2$. Basis and test functions satisfy

$$
\hat{\psi}_{0}(\zeta)=1, \quad \text { and } \quad \hat{\psi}_{m}(\zeta)=\zeta^{m}-c_{m} \quad \text { for } \quad m=1, \cdots, d_{P}
$$

were $d_{P}$ is the maximum degree of the polynomials used and constants $c_{m}$ ensure that $\psi_{m, k}(x, t)=\hat{\psi}_{m}(\zeta)$ has zero mean in $\hat{K}$ for $m \geq 1$.

Taking $d_{P}=1$, we approximate $h$ and $w$ by its mean and its slope:

$$
h_{h}(x, t)=\bar{h}_{k}+\hat{h}_{k} \psi_{1, k}(x, t) \quad \text { and } \quad w_{h}(x, t)=\bar{W}_{k}+\hat{W}_{k} \psi_{1, k}(x, t)
$$

with $\bar{U}_{k}=\hat{U}_{0, k}$, and likewise for $u$ and $v$. Alternatively,

$$
U_{h}(x, t)=\bar{U}_{k}+\hat{U}_{k} \psi_{1, k}(x, t)+\hat{U}_{2, k} \psi_{1, k}(x, t)
$$

with $h_{2, k}=0,\left(m_{u}\right)_{2, k}=\hat{h}_{k} \hat{u}_{k}$ and $\left(m_{v}\right)_{2, k}=\hat{h}_{k} \hat{v}_{k}$ These relations for $m_{u}$ and $m_{v}$ follow by projection and ensure that $h, m_{u}$ and $m_{v}$ are all zero when the depth $h$ reaches zero. Since elements are allowed to move, we define

$$
d\left|K_{k}\right| / d t=d x_{k, R} / d t-d x_{k, L} / d t, \quad d x_{k, R} / d t \equiv V_{k, R}, \quad \text { and } \quad d x_{k, L} / d t \equiv V_{k, L} .
$$

Since $\bar{W}_{k}$ and $\hat{W}_{k}$ are arbitrary, we obtain the following equations for the mean and fluctuating part after substituting (3.6) into (3.3), using (3.8), and introducing the numerical flux $\tilde{F}$ :

$$
\begin{aligned}
\frac{d}{d t}\left(\left|K_{k}\right| \bar{U}_{k}\right)= & -\left[\tilde{F}_{V}\left(U\left(x_{k, R}^{-}\right), U\left(x_{k+1, L}^{+}\right)\right)\right. \\
& \left.-\tilde{F}_{V}\left(U\left(x_{k-1, R}^{-}\right), U\left(x_{k, L}^{+}\right)\right)\right]+\frac{\left|K_{k}\right|}{2} \int_{-1}^{1} S\left(U_{h}, \zeta, t\right) \mathrm{d} \zeta \\
\frac{d \hat{U}_{k}}{d t}= & -\frac{3}{\left|K_{k}\right|}\left[\tilde{F}\left(U\left(x_{k, R}^{-}\right), U\left(x_{k+1, L}^{+}\right)\right)\right. \\
& \left.+\tilde{F}\left(U\left(x_{k-1, R}^{-}\right), U\left(x_{k, L}^{+}\right)\right)\right]+\hat{U}_{k}\left(V_{k, R}-V_{k, L}\right) /\left|K_{k}\right| \\
& +2 \hat{U}_{2, k}\left(V_{k, R}+V_{k, L}\right) /\left|K_{k}\right|+\frac{3}{\left|K_{k}\right|} \int_{-1}^{1} F \mathrm{~d} \zeta+\frac{3}{2} \int_{-1}^{1} S \zeta \mathrm{d} \zeta
\end{aligned}
$$

where $\tilde{F}_{V}\left(U_{l}, U_{r}\right)$ is a numerical flux representing $F(U)-V U$, which reduces to $\tilde{F}\left(U_{l}, U_{r}\right)$ when the nodal velocity $V=0$. The integrals of functions of $h, u$ and $v$ 
are approximated with a Gaussian quadrature. Time discretization can be done, for example, with the third-order Runge-Kutta method of Shu and Osher (1989), as used in the tests in $\S 6$.

For hyperbolic systems, numerical fluxes are often based on the exact or approximate Riemann problem at the nodes of the elements. It turns out that it is difficult to guarantee numerical stability near or at free boundaries where the depth is small or zero, because small errors in the numerical flux can lead to negative depths. We present and analyze the HLLC numerical flux in $\S 4$ and $\S 5.1$.

\section{HLLC Flux}

As noted above, since $U$ is discontinuous across element nodes, we need to approximate the flux $F(U)$ by a numerical flux $\tilde{F}(U)$. Similarly, we approximate $F_{V}=F(U)-V U$ by a numerical flux $\tilde{F}_{V}(U)$. In this section we describe one such approximation, the HLLC flux. In $\S 5.1$, we show that, when used in conjunction with a forward Euler time discretization, this approximation has the desirable property of keeping the mean depth in each element non-negative. In our approximation the depth $h=\bar{h}+\zeta \hat{h}$. Dry patches emerge when the depth becomes zero in a flooded region. Hence it is undesirable to enforce $h$ to be positive. Instead, the slope information is used to indicate where dry regions emerge, that is, when $|\hat{h}| \leq \bar{h}$.

Following Godunov, a numerical flux can be devised as follows: approximating the variable $U$ adjacent to a point by the constant values $U_{l}$ and $U_{r}$ immediately left and right of a node, one obtains a Riemann problem which can be solved exactly. However, since an exact Riemann solver is numerically expensive, the approximate HLLC solver developed by Toro et al. (1994) is used instead. Next, we briefly summarize the HLLC flux for later use.

First, consider the Riemann problem

$$
\partial_{t} U+\partial_{x} F(U)=0
$$

with constant initial left and right states $U_{l}$ and $U_{r}$, respectively. We are seeking an expression for $F-V U$ on either side of the space-time cell edge, which velocity we denote by $V$, such that a communication between the elements is properly established. In the Riemann problem for the shallow-water equation (Toro, 1999), we can distinguish four situations without dry patches where either $P_{l} \neq 0, P_{r} \neq 0$ or $u_{l}+c_{l}>u_{r}-c_{r}$ with $c=\int^{h} a(w) / w \mathrm{~d} w=2 \sqrt{g h}$ and gravity-wave speed $a=\sqrt{\partial P / \partial h}=\sqrt{g h}$, and three more situations when drying is possible. Drying occurs where $h \downarrow 0$.

Consider the element boundary $x^{\prime}=x_{k+1}(t)-x_{k+1}\left(t^{n}\right)=V\left(t^{n}\right) \tau$ for $0<\tau=$ $t^{n+1}-t^{n}<\Delta \tau$, which moves with speed $V$. The (discontinuous) values $U_{h}$, immediately left and right of this node are $U_{l}$ and $U_{r}$, respectively. In the flooded case, the HLLC approach assumes that there are four constant states from left to right $U_{l}, U_{l}^{*}, U_{r}^{*}, U_{r}$, separated at $\left(x-x_{k+1}\right) /\left(t-t^{n}\right)=S_{l}, S_{m}, S_{r}$. We integrate (4.1) over two control volumes ODCE and OABE to the left and right of the space-time node $x^{\prime}=0$, see Fig. 1, for the four cases (i) $S_{l}<V, S_{r}>V, S_{m}>V$; (ii) $S_{l}<V, S_{m}<V, S_{r}>V$; 
(iii) $S_{l}<V, S_{m}<V, S_{r}<V$; and iv) $S_{l}>V, S_{m}>V, S_{r}>V$. Subsequently, we calculate $F-V U$ along the point $x_{k+1}(t)$ as the average of the contribution on either side. After using Gauss' theorem in space and time on (4.1), we obtain (see Van der Vegt and Van der Ven, 2002):

$$
\begin{aligned}
\tilde{F}_{V}^{\text {hllc }}\left(U_{l}, U_{r}\right)= & \frac{1}{2 \Delta t}\left(\int_{\mathcal{S}_{-}}\left(F-V U_{h}\right) \bar{n}_{-K} \mathrm{~d} l_{-}+\int_{\mathcal{S}_{+}}\left(F-V U_{h}\right) \bar{n}_{+K} \mathrm{~d} l_{+}\right) \\
= & \frac{1}{2}\left\{F_{l}+F_{r}-V\left(U_{l}+U_{r}\right)-\left(\left|S_{l}-V\right|-\left|S_{m}-V\right|\right) U_{l}^{*}+\right. \\
& \left.\quad\left(\left|S_{r}-V\right|-\left|S_{m}-V\right|\right) U_{r}^{*}+\left|S_{l}-V\right| U_{l}-\left|S_{r}-V\right| U_{r}\right\},
\end{aligned}
$$

where $F_{l, r}=F\left(U_{l, r}\right), \bar{n}_{ \pm}$are outward pointing normal vectors in space time along the node $x_{k+1}(t)$, and $d l_{ \pm}$are pieces along the boundary $S_{ \pm}$of the area left and right of the node $x_{k+1}(t)$. The intermediate states $U_{l, r}^{*}$ and speed $S_{m}$ follow by using (Batten et al., 1997) a) the intermediate one star state, $\tilde{U}^{*}$, emerging from the HLL approach, and $b$ ) the Rankine-Hugoniot relations:

a) Using the HLL approach with one intermediate state $\tilde{U}^{*}$ to determine $S_{m}$, and assuming that $u_{l}^{*}=u_{r}^{*}=\tilde{u}^{*}=S_{m}$, we find that

$$
\tilde{u}^{*}=S_{m}=\frac{P_{l}-P_{r}+h_{r} u_{r}\left(S_{r}-u_{r}\right)-h_{l} u_{l}\left(S_{l}-u_{l}\right)}{h_{r}\left(S_{r}-u_{r}\right)-h_{l}\left(S_{l}-u_{l}\right)},
$$

and it may be checked that $P^{*}=P_{l}^{*}=P_{r}^{*}=P_{l}+h_{l}\left(S_{l}-u_{l}\right) /\left(S_{m}-u_{l}\right)$ as it should be for contact discontinuities with speed $S_{m}$. By retracing the derivation for $S_{m}$ we find that $S_{m}=S_{r}=S_{l}$ when $h_{r}\left(S_{r}-u_{r}\right)-h_{l}\left(S_{l}-u_{l}\right)=0$. The latter can happen when a dry patch is about to emerge.

b) By using the Rankine-Hugoniot relations for $\partial_{t} U+\partial_{x} F=0$ we find

$$
\left(S_{l, r}-S_{m}\right) U_{l, r}^{*}=\left(S_{l, r}-u_{l, r}\right) U_{l, r}+\left(\begin{array}{c}
0 \\
P^{*}-P_{l, r} \\
0
\end{array}\right)
$$

with

$$
P^{*}=P_{l, r}^{*}=P_{l, r}+h_{l, r}\left(S_{l, r}-u_{l, r}\right)\left(S_{m}-u_{l, r}\right)
$$

and velocities $u_{l, r}$. By combining (4.2) and (4.4) we can deal with the cases $S_{l, r}=S_{m}$ in order to avoid a division by zero. Handling the latter is important when $h=0$ at one of the nodes of an element.

Finally, the wave speeds are estimated based on the left and right moving rarefaction wave speeds

$$
S_{l}=\min \left(u_{l}-a_{l}, u_{r}-a_{r}\right), \quad S_{r}=\max \left(u_{l}+a_{l}, u_{r}+a_{r}\right),
$$


respectively. When $S_{l}>V$ the flux simplifies to $F_{l}-V U_{l}$ and when $S_{r}<V$ to $F_{r}-V U_{r}$, i.e. the upwind cases. When we choose a Lagrangian grid velocity the choice $V=S_{m}$ is appropriate since

$$
\lim _{V \rightarrow S_{m}} \tilde{F}_{V}^{h l l c}\left(U_{l}, U_{r}\right)=\left(0, P^{*}, 0\right)^{T},
$$

and $P^{*}=P_{r}$ if $h_{l}=0$ and $u_{r}=S_{m}$, or $P^{*}=P_{l}$ if $h_{r}=0$ and $u_{l}=S_{m}$.

\section{$5 \quad$ Flooding and Drying}

\section{Definition 1: Fluid patches}

The fluid is divided into one or more disjoint patches of fluid in a bounded region, see Fig. 2. In one dimension, each patch has a left and right boundary. The boundary conditions at the external boundaries are, for example, (prescribed) in- or outflow conditions (depending on the characteristics), and no flow through moving or fixed walls.

In one dimension a dry-wet boundary is demarcated by a particle on the left or right of the patch. There are three situations to distinguish: i) a patch of fluid moves along with a dry-wet boundary on the left, on the right, or at both sides; ii) a patch of fluid breaks up into two patches in which case we may have to define two new particles; and iii) two patches of fluid merge into one patch of fluid.

\section{Definition 2: Free-boundary elements}

The elements of a patch consist of Eulerian fixed elements and mixed EulerianLagrangian free-boundary elements. The Eulerian fixed elements of $\Omega_{f}$ coincide with a subset of underlying fixed elements of the computational domain $\Omega$.

A free, dry-wet boundary on the right of a patch is modeled with a free-boundary element whose right node moves with the flow, see Fig. 3. At this right node of the free-boundary element the depth is generally zero. The left node of this free-boundary element is fixed.

When the depth is zero at this right node $k=\mathrm{ri}$, we constrain the linear representation $h_{k}=\bar{h}_{k}+\hat{h}_{k} \zeta$ to be zero at $\zeta=1$ such that $\hat{h}_{\mathrm{ri}}=-\bar{h}_{\mathrm{ri}}$. From the mean part of (3.9), we find the equations of motion for $\bar{h}_{r i}$

$$
\left|K_{\mathrm{ri}}\right| \frac{d \bar{h}_{\mathrm{ri}}}{d t}=\tilde{F}_{h}\left(U_{\mathrm{ri}-1, R}, U_{\mathrm{ri}, L}\right)-\bar{h}_{\mathrm{ri}} V_{R}
$$

with $V_{R}=d x_{\mathrm{ri}, R} / d t$ and $V_{L}=0$. The subscript in $\tilde{F}_{h}$ denotes that we consider the depth component $h u$ of the flux (4.2) with $V_{L}=0$. Likewise for a free-boundary element on the left $k=$ le, and we constrain $\hat{h}_{\mathrm{le}}=\bar{h}_{\mathrm{le}}$. From (3.9), we find the equations of motion for $\bar{h}_{\mathrm{le}}$

$$
\left|K_{\mathrm{le}}\right| \frac{d \bar{h}_{\mathrm{le}}}{d t}=-\tilde{F}_{h}\left(U_{\mathrm{le}, R}, U_{\mathrm{le}+1, L}\right)+\bar{h}_{\mathrm{le}} V_{L}
$$


with $V_{L}=d x_{\mathrm{le}, L} / d t$ and $V_{R}=0$. The discretization for the other variables remains unconstrained, which formally ensures second-order accuracy for the velocity and hence the advancement of the free boundary.

\section{Definition 3}

Define the Heaviside function $\Theta(x)$ with $\Theta(x)=0$ when $x<0$ and $\Theta(x)=1$ for $x \geq 0$, and denote by $S_{m L}$ the $S_{m}$-velocity at the left node of an element, and so forth.

\subsection{Time-step criterion for the mean depth}

Using an forward Euler time step scheme, we next show that the mean depth remains positive for a suitably restricted time step.

Proposition 5.1. We consider the following assumptions:

A1: Use the discontinuous Galerkin finite-element discretization (3.9), either with a mean only or with both mean and slope information, together with the HLLC flux defined in (4.2)-(4.6).

A2: Use a forward Euler time stepping scheme to integrate the continuity equation $\partial_{t} h+\partial_{x}(h u)=0$ from time $t^{n}$ to time $t^{n+1}$.

A3: Let the depth at time $t^{n}$ in element $K_{k}$ be $h_{k}^{n}$ with $h_{k, L}^{n}$ and $h_{k, R}^{n}$ the values at the left and right nodes of this element. For interior (flooded) elements we have $h_{k, L}^{n}>0, h_{k, R}^{n}>0$ and $V_{R}=V_{L}=0$. For a free boundary element on the left we have $h_{k, L}^{n}=0, h_{k, R}^{n}>0$ and $V_{R}=0$. Likewise, for a free boundary element on the right we have $h_{k, L}^{n}>0, h_{k, R}^{n}=0$ and $V_{L}=0$.

Given assumptions A1-A3 and the definitions 1-3, the mean depth $\bar{h}^{n+1}$ at the next time step is positive provided the time step is less than the minimum value of the elemental time step $\Delta t_{k}$ in element $K_{k}$ is taken. This elemental time step $\Delta t_{k}$ is defined in (5.6).

Proof. Consider the forward Euler time integration for the mean depth in the discontinuous Galerkin finite-element discretization:

$$
\begin{aligned}
\bar{h}_{k}^{n+1}= & \bar{h}_{k}^{n}-\Delta t\left[\tilde{F}_{V, h}\left(U_{k, R}^{n}, U_{k+1, L}^{n}\right)-\tilde{F}_{V, h}\left(U_{k-1, R}^{n}, U_{k, L}^{n}\right)\right] /\left|K_{k}^{n}\right|+ \\
& \Delta t\left(V_{L}^{n}-V_{R}^{n}\right) \bar{h}_{k}^{n} /\left|K_{k}^{n}\right|,
\end{aligned}
$$

where $\bar{h}_{k}^{n+1}$ is the depth at the next time step and $\bar{h}_{k}^{n}$ the depth at the previous time step in element $k$. Define the shorthand $S_{l}^{V}=S_{l}-V$, et cetera. The subscripts in 
$\tilde{F}_{V, h}$ denotes that we consider the depth component $h u$ of the flux $(4.2)$, i.e.

$$
\begin{aligned}
2 \tilde{F}_{V, h}= & u_{l} h_{l}+u_{r} h_{r}-\left(\left|S_{l}^{V}\right|-\left|S_{m}^{V}\right|\right) h_{l}^{*}+\left(\left|S_{r}^{V}\right|-\left|S_{m}^{V}\right|\right) h_{r}^{*}+ \\
& \left|S_{l}^{V}\right| h_{l}-\left|S_{r}^{V}\right| h_{r}-V\left(h_{l}+h_{r}\right) \\
= & \left(\left(S_{l}-S_{m}+\left|S_{l}^{V}\right|-\left|S_{m}^{V}\right|\right) u_{l}+\left(\left|S_{m}^{V}\right|-V\right) S_{l}+\left(V-\left|S_{l}^{V}\right|\right) S_{m}\right) \frac{h_{l}}{S_{l}-S_{m}}+ \\
& \left(\left(S_{r}-S_{m}-\left|S_{r}^{V}\right|+\left|S_{m}^{V}\right|\right) u_{r}-\left(\left|S_{m}^{V}\right|+V\right) S_{r}+\left(V+\left|S_{r}^{V}\right|\right) S_{m}\right) \frac{h_{r}}{S_{r}-S_{m}} .
\end{aligned}
$$

The flux (5.4) at a node reduces to the following four cases

$$
\tilde{F}_{V, h}=\left\{\begin{array}{lcc}
h_{r}\left(u_{r}-V\right)<0 & \text { if } & S_{l}^{V}<S_{m}^{V}<S_{r}^{V}<0 \\
h_{r}\left(V-S_{m}\right)\left(u_{r}-S_{r}\right) /\left(S_{r}-S_{m}\right)<0 & \text { if } & S_{l}^{V}<S_{m}^{V}<0 \wedge S_{r}^{V}>0 \\
h_{l}\left(u_{l}-V\right)>0 & \text { if } & 0<S_{l}^{V}<S_{m}^{V}<S_{r}^{V} \\
h_{l}\left(S_{m}-V\right)\left(u_{l}-S_{l}\right) /\left(S_{m}-S_{l}\right)>0 & \text { if } & S_{l}^{V}<0 \wedge 0<S_{m}^{V}<S_{r}^{V}
\end{array}\right.
$$

in which we used the relation (4.6) to determine these inequalities.

The elemental time step

$$
\begin{aligned}
\Delta t_{k}=\bar{h}_{k}^{n} \mid & K_{k} \mid / \max \left(\left(V_{R}-V_{L}\right) \bar{h}_{k}+h_{r L}\left(V_{L}-u_{r L}\right) \Theta\left(-S_{m L}\right) \Theta\left(-S_{r L}\right)\right. \\
& +h_{r L} \frac{\left(V_{L}-S_{m L}\right)\left(S_{r L}-u_{r L}\right)}{\left(S_{r L}-S_{m L}\right)} \Theta\left(-S_{m L}\right) \Theta\left(S_{r L}\right) \\
& -h_{l L}\left(u_{l L}-V_{L}\right) \Theta\left(S_{l L}\right) \Theta\left(S_{m L}\right) \\
& -h_{l L} \frac{\left(S_{m L}-V_{L}\right)\left(u_{l L}-S_{l L}\right)}{\left(S_{m L}-S_{l L}\right)} \Theta\left(-S_{l L}\right) \Theta\left(S_{m L}\right) \\
& +h_{l R} \frac{\left(S_{m R}-V_{R}\right)\left(u_{l R}-S_{l R}\right)}{\left(S_{m R}-S_{l R}\right)} \Theta\left(S_{m R}\right) \Theta\left(-S_{l R}\right) \\
& \left.+h_{l R}\left(u_{l R}-V_{R}\right) \Theta\left(S_{m R}\right) \Theta\left(S_{l R}\right)\right) \\
& -h_{r R} \frac{\left(S_{m R}-V_{R}\right)\left(u_{r R}-S_{r R}\right)}{\left(S_{r R}-S_{m R}\right)} \Theta\left(-S_{m R}\right) \Theta\left(S_{r R}\right) \\
& \left.-h_{r R}\left(V_{R}-u_{r R}\right) \Theta\left(-S_{m R}\right) \Theta\left(-S_{r R}\right), 0\right) .
\end{aligned}
$$

All terms on the right-hand-side in (5.6) are evaluated at time $t^{n}$.

We obtain (5.6) and the time step restriction $\Delta t<\Delta t_{k}$ by using the result (5.5) in (5.3) and requiring that $\bar{h}_{k}^{n+1}>0$. Note that each term in the denominator on the right-hand-side of (5.6) is positive. The sign in front of a term in this bracket thus signals whether it yields a positive or negative contribution to the denominator.

Procedure 1: Merging and splitting of elements

In order to maintain the mostly Eulerian nature of the numerical scheme, i) we split a free-boundary element when it becomes too large, and ii) merge a free-boundary 
element with its neighbor when the former becomes too small. Therefore, the number of elements in a patch with at least one free boundary may change over time. Due to the local nature of discontinuous Galerkin finite-element methods, this update can be handled locally.

The element-splitting process (which operationally is performed when a freeboundary element becomes larger than, say, the size of the local underlying fixed element $K_{k}$ plus 0.6 times the size of the overflown adjacent fixed element) is quite simple: the part of the free-boundary element entirely between two of the fixed nodes is made a regular element, and the remainder the new free-boundary element [see Fig. 4a]. For the constrained linear basis function, no information is lost in this process. We determine the new values simply by projection. Mean and slope values of the depth and the velocity can then preserved in this splitting process. This operation is not performed when there is a front nearby (and a patch merger - see below-is imminent).

In the element-merging process (performed operationally when the size of an edge element falls below, say, 0.4 times that of the local regular element), we construct a new free (i.e. "triangular") element from the old free-boundary element and its neighbor, preserving the integral of $h_{h}, u_{h}, v_{h}$, and the location of the original front [see Fig. 4b]. For the linear basis function at the free boundary, the slope information $\hat{h}_{k}$ of the neighboring element is constrained. Only the mean value of $h$ is thus preserved.

\section{Procedure 2: Merging and splitting of patches}

In addition to element merging and splitting discussed above, patches may also merge and split. When the depth of the fluid becomes zero in the interior of a patch or when a splitting criterion is met, the patch is split into two patches with free-boundary conditions at the splitting point. In the absence of source terms $S$, the splitting criterion derives optimally from a Riemann splitting criterion for a flat bottom case, that is, at an edge splitting occurs when $u_{l}+c_{l} \leq u_{r}-c_{r}$ or approximately since $c_{l, r} \ll 1$, when $u_{l} \leq u_{r}$ (see, e.g., Smoller, 1994; and Toro, 1999). Alternatively, the Riemann problem may be considered for a locally uniform bottom slope. When fronts from two patches meet, we merge the patches into a single patch. For computational reasons, patches are constrained to have a minimum of two elements.

It is easy to see that, due to our linear basis functions, the depth of the fluid must first become zero at a node before it does in the interior of an element. Thus, given sufficient temporal resolution as guaranteed by the time-step criterion for the depth, patch splitting always occurs at one or two nodes of an element for linear or constant basis functions, respectively. When this happens, the (regular) elements bordering the node(s) are made free-boundary elements having the same length as the underlying regular elements, preserving the integral of $U$ [see Fig. 5].

When the free boundaries of two patches meet or overlap after a full time step, the patches are merged: the free-boundary elements are converted into regular elements, preserving as much of the original information as possible. Due to the finite temporal resolution in practice, we perform the patch merging procedure when two free-boundary elements have actually overlapped. There are several scenarios for this 
overlap, which can be simplified if we split the free-boundary elements before merging the patches. After the boundary-element splitting, we are left with two possibilities: (a) the more generic case where the free boundary elements overlap within one regular element [see Fig. 6a], and (b) the less generic case where the free-boundary elements overlap within two regular elements [see Fig. 6b].

A patch is not split if a new patch would consist of only one element.

A note on implementation: the set of patches (whose number should be small in normal operation) is implemented as a linked list, each of which contains information about the boundary conditions, the location of the boundaries, of the neighboring patches (if any), and the free-boundary elements (if any).

Definition 4

The preliminary time step $\Delta t_{p}$ is the minimum over all elements of each time step associated with the maximum wave speed in element $K_{k}$

$$
\left|K_{k}\right| / \min \left(\left|u_{k}\right|+\sqrt{g h_{k}}\right),
$$

and the time step estimate $\Delta \tilde{t}_{k}$ in each left and right free-boundary element:

$$
\begin{aligned}
\Delta \tilde{t}_{k}< & \left|K_{k}\right| /\left(\max \left(V_{R}-2 u_{r L} \Theta\left(-S_{m L}\right), 0\right) \max \left(-S_{r L}, 0\right)+\right. \\
& \left.\max \left(V_{R}+2 \frac{\left|S_{m L}\right|\left(S_{r L}-u_{r L}\right)}{\left(S_{r L}-S_{m L}\right)} \Theta\left(-S_{m L}\right), 0\right) \max \left(S_{r L}, 0\right)\right) \\
\Delta \tilde{t}_{k}< & \left|K_{k}\right| /\left(\max \left(-V_{L}+2 \frac{\left|S_{m R}\right|\left(u_{l R}-S_{l R}\right)}{\left(S_{m R}-S_{l R}\right)} \Theta\left(S_{m R}\right), 0\right) \max \left(-S_{l R}, 0\right)+\right. \\
& \left.\max \left(-V_{L}+2 u_{l R} \Theta\left(S_{m R}\right), 0\right) \max \left(S_{l R}, 0\right)\right)
\end{aligned}
$$

respectively. In order to derive (5.8) and (5.9), we used the inequalities $h_{k, R} \leq 2 \bar{h}_{k}$ and $h_{k, L} \leq 2 \bar{h}_{k}$ in (5.6) and ignored the terms with a minus sign in the denominator of the right-hand-side of (5.6).

\subsection{Stepping forward in time}

Corollary 5.2. We consider the following assumptions:

A4: For all interior elements, depth and velocity are approximated by a mean and slope, as follows, $h=\bar{h}_{k}+\zeta \hat{h}_{k}, u=\bar{u}+\zeta \hat{u}$ and $v=\bar{v}+\zeta \hat{v}$. Define $m_{u}=h u=$ $\bar{m}_{u}+\zeta \hat{m}_{u, 1}+\left(\zeta^{2}-1 / 3\right) \hat{m}_{u, 2}$ and likewise for $m_{v}=h v$. Using a weak formulation for $m_{u}=h u$ and $m_{v}=h v$, we then obtain

$$
\bar{u}=\frac{3 \bar{m}_{u} \bar{h}-\hat{m}_{u, 1} \hat{h}}{3 \bar{h}^{2}-\hat{h}^{2}}, \quad \hat{u}=\frac{3\left(\hat{m}_{u, 1} \bar{h}-\bar{m}_{u} \hat{h}\right)}{3 \bar{h}^{2}-\hat{h}^{2}}, \quad \hat{m}_{u, 2}=\hat{h} \hat{u}
$$

and likewise for $\bar{v}$ and $\hat{v}$.

A5: For free boundary elements, depth and velocity are approximated by $h_{k}=\bar{h}_{k}(1 \pm \zeta)$ at left and right free boundary elements, respectively. 
A6: In a free boundary element on the left $V_{L}=u_{k, L}$. In a free boundary element on the right $V_{R}=u_{k, R}$.

A7: The time step $\Delta t$ is the minimum of $\Delta t_{p}$ in definition 4 and time steps $\Delta \hat{t}_{k}$ for interior elements. Here $\Delta \hat{t}_{k}$ is the local time step for which $\bar{h}_{k}^{n+1} \pm \hat{h}_{k}^{n+1}=0$, in which $\bar{h}_{k}^{n+1} \pm \hat{h}_{k}^{n+1}$ follows from the discontinuous Galerkin discretization (3.9) for the continuity equation as

$$
\begin{aligned}
\bar{h}_{k}^{n+1} \pm \hat{h}_{k}^{n+1}= & \bar{h}_{k}^{n} \pm \hat{h}_{k}^{n}-\frac{\Delta t}{\left|K_{k}^{n}\right|}\left((1 \pm 3) \tilde{F}_{h}^{h l l c}\left(U_{k, R}^{n}, U_{k+1, L}^{n}\right)+\right. \\
& \left.(-1 \pm 3) \tilde{F}_{h}\left(U_{k-1, R}^{n}, U_{k, L}^{n}\right)\right) \pm \frac{2 \Delta t}{\left|K_{k}^{n}\right|}\left(3 \bar{h}_{k}^{n} \bar{u}_{k}^{n}+\hat{h}_{k}^{n} \hat{u}_{k}^{n}\right)
\end{aligned}
$$

with $\tilde{F}_{h}=\tilde{F}_{V=0, h}$ given in (5.5). Note that $V_{R}=V_{L}=0$ for interior elements.

A8: When the depth of an interior element $K_{k}$ falls dry after the time step $\Delta \hat{t}_{k}$ at node $x_{k}$ or $x_{k+1}$, the patch to which $K_{k}$ belongs is split and left and right free boundary elements $K_{k-1}$ and $K_{k}$ or $K_{k}$ and $K_{k+1}$ emerge.

Given definitions 1-4, procedures 1 and 2, and assumptions 1-8 the shallow-water equations are integrated forward in time with a discontinuous Galerkin finite-element method formally first order in time and second order in space under preservation of non-negative water depth.

Proof. By construction.

Remarks:

(i) The proposition and corollary extend to the third-order Runge-Kutta scheme by repeating the proposition for every intermediate stage with intermediate depth $h^{(i)}$ assuming subsequently that $\bar{h}^{(i)} \pm \hat{h}^{(i)} \geq 0$ and $\bar{h}^{(i)}>0$. Unfortunately, a smaller time step for $h^{(i)}$ may require a restart at $t^{n}$.

(ii) The conditions in remark (i) are ensured by limiting $m$ and $h$ to zero whenever $h<0$ at one side of an interior element at an intermediate stage when the multi-stage third-order Runge-Kutta method is used. This limiting is necessary because we are unable to find the time step at which a dry patch emerges in the multi-stage time discretization, in contrast to the forward Euler case.

(iii) The proof shows that the maximum time step allowed per element is most easily implemented numerically in parallel with the determination of the fluxes.

(iv) For a patch that consists of only two elements the velocities left and right of a patch are set equal in order to avoid very thin and small patches. When this situation emerges, the dynamics has essentially reached the subgrid scales. The numerical model is then no longer expected to be accurate.

(v) The proposition and corollary are in principle valid for any system discretized in a similar way and with a one-dimensional continuity equation. Hence, it is not restricted to the shallow-water case, but also valid, for example, for the compressible Euler equations by replacing $h$ by a density $\rho$. 


\section{Verification}

In the numerical verification, we consider the dam break problem, the drying Riemann problem over a flat bottom, the parabolic bowl solution (Young, 1986), the CarrierGreenspan solution (Carrier and Greenspan, 1958) and the Peregrine-Williams solution (Peregrine and Williams, 2001). These exact solutions are summarized in Appendix A.

In the verification cases, the numerical solution is compared with the exact solution. We use the $L^{2}$-errors

$$
\begin{aligned}
L^{2}(u, h) & =\left(\int_{\Omega_{f}}\left(u_{\text {numerical }}-u_{\text {exact }}\right)^{2}+\left(h_{\text {numerical }}-h_{\text {exact }}\right)^{2} \mathrm{~d} x / L_{f}\right)^{1 / 2} \\
L^{2}(m, h) & =\left(\int_{\Omega_{f}}\left(U_{\text {numerical }}-U_{\text {exact }}\right)^{2} \mathrm{~d} x / L_{f}\right)^{1 / 2}
\end{aligned}
$$

with $L_{f}$ the flooded part of the computational domain, and the $L^{\infty}$-error, the maximum difference between the numerical and exact solution. When possible and useful, the difference between the exact and numerical solution of the frontal position, and the error in the break up time of a patch of fluid is calculated as well.

Dimensionless, scaled equations have been used, effectively taking $g=1$. Shallow slopes of 1:20 or 1:100 emerging in the dimensional problem, $c f$. the shallowness assumption, therefore often appear as slopes of order one in our dimensionless examples (see the scalings in, e.g., Brocchini and Peregrine, 1996; and Peregrine and Williams, 2001). The total number of fixed finite elements across the computational domain is stated in each case below. In the actual computation, only the flooded (fixed and free boundary) elements are used in action.

\subsection{Riemann solutions}

A summary of the solutions to the Riemann problem for the shallow-water equations can be derived from Smoller (1994) or Toro (1999).

\subsubsection{Dam break}

Consider a dam break problem with an initial condition $h(x, 0)=H_{0}$ for $x-x_{0}<0$ and zero elsewhere, and $u(x, 0)=0$. Since the initial condition is discontinuous, we adapted our boundary treatment by using both slope and mean as variable as long as the depth at the front is positive, while the speed of the front is the prediction $u_{l}+c_{l}$ with $c=2 \sqrt{g h}$ for the Riemann problem with one side a dry bed. Otherwise, when the depth has become zero at the front, the free-boundary treatment explained before is used.

The numerical and exact solutions are displayed in Fig. 7. From Table 1, we conclude that the solution converges at order $\sim 0.7$ in the $L^{2}(u, h)$-norm, at order $\sim 0.65$ in the position of the front, while it is of order 0.9 for the $L^{2}(m, h)$-norm and 0.4 in the $L^{\infty}$-error. 
Both the dam break problem and the next case in which a dry patch emerges are special. These solutions start from a discontinuity, and then the front moves over the horizontal topography such that the depth becomes tangent to the bed in a quadratic manner near the free boundary on the right of a patch, i.e. $h \propto\left(x_{R}(t)-x\right)^{2}$. The depth and speed at the front in our discretization, however, are approximated with linear polynomials. Higher-order (constrained) polynomial approximations tangent to the bed may be required to improve the approximation of $m_{u}$ and $h$ in the free boundary element. In addition, the solution does not have continuous derivatives for $t>0$, which may also impede second-order accuracy. In contrast, in the other cases which are considered with topography, the free surface intersects the boundary at a finite angle, and the representation by linear polynomials appears sufficient even when the actual solution is quadratic (but not tangent to the bed).

\subsubsection{Drying}

A dry patch occurs in Riemann problems when the constant initial data at $t=0$ meet the drying criterion $c_{l}+c_{r}-u_{r}+u_{l}<0$. Two expansion waves then propagate away from a dry patch, which immediately appears at $t \downarrow 0$.

The numerical results in Table 2 and Fig. 8 show that the accuracy is of order 0.9 for $L^{2}(m, h)$, while the $L^{2}(u, h)$ - and $L^{\infty}$-errors are fixed. The initial conditions are chosen such that a small dry patch develops. The numerical solution develops this dry patch initially, but due to the numerical dissipation inherent in the HLLC flux the patch incorrectly floods again thereafter. As in the dam break problem, the velocity and depth are constant and linear in the numerical solution, while being linear and quadratically tangent to the bed in the exact solution. Again, higher-order constrained polynomials at the free boundary may be required to (partially) remedy this mismatch.

\subsection{Parabolic bowl}

The results in Table 3 and Fig. 9 show that the numerical accuracy is of order 2 . The parabolic test solution is thus sufficiently smooth so that the numerical results converge to the expected second-order accuracy. The difference of the exact and numerical position of the free boundary at a fixed time is not such a good indicator (see $\epsilon_{x_{b}}$ in Table 3), perhaps because the solution and its error are oscillatory or because the element splitting process (temporarily and intermittently) reduces accuracy (which is not visible in the graph of $x_{b}(t)$ versus $\left.t\right)$. Nevertheless, this difference converges as well, but slower. Note that the free surface in the parabolic bowl solution intersects the topography at a finite angle, in contrast to the previous two solutions.

\subsection{Carrier-Greenspan}

The numerical results are given in Table 4 and Fig. 10. The numerical accuracy in the interior is about order 1 for the $L^{2}$-norms and around order 0.4 for the $L^{\infty}$-norm 
and about order 1.2 for the error in the frontal position. The plot of the height field $h(x, t)$ shows clearly that some accuracy is lost where the gradient becomes very steep or infinite, which coincides with the region where the Jacobian of the transformation becomes small. The latter may explain why the convergence is not optimal, i.e. of order 2 .

\subsection{Peregrine-Williams}

Peregrine and Williams (2001) derived an analytical solution for a dam break problem of a shallow-water layer on a steep slope of a dike. Eventually, the water reaches the top of the dike and falls down. The top of the dike defines a critical point in the flow. Peregrine and Williams (2001) scaled the shallow-water equations, which form is equivalent to shallow-water equations either on a shallow slope with a horizontal coordinate $x$ or on a steep slope in which coordinate $x$ follows the slope. Here, we used the former, with a horizontal coordinate $x$.

A numerical simulation with a resolution of 200 elements and no extra numerical dissipation shows the numerical (solid lines) and exact (dashed lines) in Fig. 11. The order of accuracy of the $L^{2}$-errors is about 1 . The accuracy of the front position is about 0.7. The prediction of the break up time converges slowly, eventually at an order 0.5 . The $L^{\infty}$-error is around order $0.3-0.4$.

\section{$7 \quad$ Run-up and Overtopping}

Finally, a complex simulation with wave breaking, run-up and overtopping is shown to display the potential of the presented numerical discretization of the shallow-water equations. The space-time plot of depth and velocity profiles in Fig. 12 depicts the time history of multiple waves steepening to bores, running up the beach, and spilling water over the top of the dike. This dike top is a critical point as in the PeregrineWilliams solution. Beyond the dike top the water rushes down in broken patches of fluid. The offshore beach slope is scaled to unity, and a wave maker introduces sinusoidal waves offshore. At the left boundary $x=0$, we specify the state $U_{l 0}$, used in the flux calculation. It is grown from rest and $h=H_{0}$ linearly in $t$ from $0<t<0.1$ to the solution for $t \geq 0.1$

$$
u_{l}(0, t)=-k A \sin (k x-\omega t) / \omega \quad h_{l}(0, t)=H_{0}-A \sin (k x-\omega t)
$$

with $H_{0}=1, k=\omega=3 \pi, A=0.07$ and initial condition $h(x, 0)=H_{0}-x$ for $x<H_{0}$ and $u(x, 0)=0$. The offshore boundary condition is implemented with the same HLLC flux scheme used at interior element nodes. Essentially depending on the characteristics, information is thus (partly) flowing in or out the domain. The landward boundary is open, such that water is allowed to vanish.

In the simulation in Fig. 12, we see initially sinusoidal waves steepen to bores when they approach the shore. After a bore has formed the slope between bores is nearly parallel to the beach topography, and the dynamics behind the last bore resembles 
the initial condition of the idealized overtopping solution of Peregrine and Williams. The evolution of each incoming bore then resembles intermittently the PeregrineWilliams solution. Due to the offshore driving of waves, multiple bores create multiple overtopping events. On the right side of the dike top, multiple patches with very shallow water rush down the slope rapidly to leave the computational domain.

\section{Conclusions}

This paper is a study of the free-boundary dynamics in one-dimensional shallow-water equations with a space discontinuous Galerkin finite-element scheme. Our numerical scheme is able to preserve the non-negative nature of the (mean) depth in combination with a free-boundary treatment in mixed Eulerian-Lagrangian elements. A variety of solutions has been considered in which the free-boundary movement is essential, including cases where free boundaries emerge or disappear.

We combined an explicit space discontinuous Galerkin finite-element scheme (Cockburn and Shu, 1998) with the HLLC flux to ensure positivity of the mean water depth in each element under certain reasonable time-step restrictions. A spatial discretization was considered which is formally second-order accurate with constant and linear polynomial basis functions, representing the mean and slope of the velocity and depth in each element. The free-boundary movement and the appearance of dry patches with zero water depth in the middle of the fluid domain has been handled by using the slope information of the depth. Finally, the robustness of the method is exemplified by a complex simulation of multiple shoaling and steepening water waves running up the seaward slope of a dike, overtopping, and then breaking up in two or more patches at the downslope side.

Detailed and varied numerical verifications show that our method is second order in smooth cases and in the interior of the domain in the absence of physical discontinuities. It reduces to order 0.7 to 1.0 in the presence of discontinuities, and at the free boundary. It reduces to order 0.5 at the front when (multiple) dry patches emerge. Riemann problems with dry patches on horizontal topography lead to numerical solutions with inaccurate predictions of the free-boundary position, because the linear polynomial approximations cannot converge well to these solutions in which the free surface is parallel to the topography at the free boundary, and because the initial condition near (emerging) free boundaries is discontinuous. The initial discontinuity can be avoided starting with a continuous profile in this Riemann problem. A discontinuity in the first derivative, however, also prevents convergence to second-order accuracy.

Local $p$-refinement combined with an asymptotic analysis at the free boundary (such that the order of the basis and test function is raised) is anticipated to (partly) remedy the accuracy loss in these Riemann problems with dry patches and raise the accuracy at the edge elements in general (see also Bokhove et al., 2003). However, the introduction of a constrained second-order basis function in these elements poses additional restrictions in order to ensure positivity of the mean depth. Patch 
splitting also reduces the accuracy. Using space-time discontinuous Galerkin finiteelement methods can possibly lead to more accurate patch splitting because the time of splitting is then known directly. The implicit nature of the space-time method and the stability of the required iterative solvers of the nonlinear algebraic problem can, however, pose drawbacks.

The extension of the flooding and drying approach to two dimensions is in progress. The HLLC flux can be extended to higher dimensions (Van der Vegt and Van der Ven, 2002), as well as proposition 5.1 on the time step criterion ensuring positivity of the mean depth in a triangulation (Bokhove, 2003). The proposal (Bokhove, 2003) is to maintain the mesh quality by updating the mesh topology by locally deforming an underlying basic, regular mesh of rectangles to the fixed and free boundaries. A triangular mesh is then defined by inserting diagonals in this (locally deformed) rectangular mesh. As in the one-dimensional mesh in Fig. 3, an ALE computational mesh is used in combination with a fixed, regular mesh. The structured fixed mesh in principle allows for a more efficient handling of the motion and upgrading of the computational mesh, as nodes are restricted to move along the rectangular faces or their diagonals of the fixed mesh only.

In addition, an implicit space-time discontinuous Galerkin finite-element method is under development for flooding and drying in two-dimensional shallow-water equations. In this approach, the use of a cut cell approach is anticipated.

Acknowledgments

O.B. gratefully acknowledges a fellowship from The Royal Dutch Academy of Arts and Sciences (KNAW). Part of the analysis and coding was done by dr. Djoko Wirosoetisno, who also made numerous useful suggestions, thanks to a visitors' stipend from the Twente Institute of Mechanics of the University of Twente. A preliminary version of the presented work is found in Bokhove and Wirosoetisno (2003). Professor J.J.W. van der Vegt provided valuable advice and comments during the course of this work.

\section{References}

Audusse, E., \& Bristeau, M.-O. (2003) 'Transport of pollutant in shallow water: a two time steps kinetic method'. ESAIM-Math. Mod. and Num. Analysis 37, 389-416.

Batten, P., Clarke, N., Lambert, C., \& Causon, D.M. (1997) 'On the choice of wavespeeds for the HLLC Riemann solver'. Siam. J. Sci. Comput. 18, 1553-1570.

Bokhove, O. (2003) 'Flooding and drying in Finite-Element Discretizations of ShallowWater Equations. Part 2: Two Dimensions'. Memorandum 1684, Mathematical Communications 2003 University of Twente, www.math.utwente.nl/publications/

Bokhove, O., \& Wirosoetisno, D. (2003) 'Drying and Wetting in Finite Element ShallowWater Flows'. Eds. G.H. Jirka \& W.S.J. Uijttewaal, Proceedings of the International Symposium on Shallow Flows, Delft University of Technology, 16-18 June 2003, Part III, 153-160.

Bokhove, O., Woods, A.W., \& Boer de, A. (2003) 'Magma Flow through Elastic-Walled Dikes'. Manuscript under internal review at Southwest Research Institute, 32 pp.

Brocchini, M., \& Peregrine, D.H., (1996) 'Integral flow properties of the swash zone and averaging'. J. Fluid Mech. 317, 241-273. 
Carrier, G.F., \& Greenspan, H.P. (1958) 'Water waves of finite amplitude on a sloping beach'. J. Fluid Mech. 4, 97-109.

Cockburn, B., Lin, S.-Y., \& Shu, C.-W. (1989) 'TVB Runge-Kutta local projection discontinuous Galerkin finite element method for conservation laws III: one dimensional systems. J. Comp. Phys. 84, 90-113.

Cockburn, B., \& Shu, C.-W. (1998) 'The Runge-Kutta discontinuous Galerkin finite element method for conservation laws V: multidimensional systems. J. Comp. Phys. 141, 199224.

Jaffre, J, Johnson, C., \& Szepessy, A. (1995) 'Convergence of the discontinuous Galerkin finite element method for hyperbolic conservation laws'. Math. Models and Methods in Appl. Sci. 5, 367-386.

Krivodonova, L., Xin, J., Remacle, J.-F., Chevaugeon, N., \& Flaherty, J.E. (2003) 'Shock detection and limiting with discontinuous Galerkin methods for hyperbolic conservation laws'. Applied Numerical Mathematics, accepted.

Morton, K.W., \& Mayers, D.F. (1994) Numerical solution of partial differential equations. CUP, Cambridge, 227 pp.

Pedlosky, J. (1987) Geophysical Fluid Dynamics. Springer, New York, 710 pp.

Peregrine, D.H., \& Williams, S.M. (2001) 'Swash overtopping a truncated plane beach'. $J$. Fluid Mech. 440, 391-399.

Perthame, B. (1999) 'An introduction to kinetic schemes for gas dynamics'. In: An introduction to recent developments in theory and numerics for conservation laws. Eds. Kröner, D., Ohlberger, M., \& Rohde, C., Springer Verlag. 1-27.

Perthame, B., \& Simeoni, C. (2001) 'A kinetic scheme for the Saint-Venant scheme with a source term'. Calcolo 38, 201-231.

Schwanenberg, D. (2003) Die Runge-Kutta-Discontinuous-Galerkin-Methode zur Lösung konvektionsdominierter tiefengemittelter Flachwasserprobleme. Ph.D. Dissertation Technischen Hochschule Aachen, 133 pp.

Shen, M.C., \& Meyer, R.E. (1963) 'Climb of a bore on a beach'. J. Fluid Mech. 16, 113-125.

Smoller, J. (1994) Shock waves and reaction-diffusion equations. Springer Verlag, New York, $632 \mathrm{pp}$.

Shu, C-W., \& Osher, S. (1989) 'Efficient implementation of essentially non-oscillatory shockcapturing schemes II', J. Comp. Phys. 83, 32-78.

Toro, E.F., Spruce, M., \& Speares, W. (1994) 'Restoration of the contact surface in the HLL-Riemann solver. Shock Waves 4, 25-34.

Toro, E.F. (1999) 'Shock capturing methods for free-surface flows', Wiley, Toronto, 309 pp.

Young, W.R. (1986) 'Elliptical vortices in shallow water'. J. Fluid Mech. 171, 101-119.

Vegt van der, J.J.W., \& Ven van der, H. (2002) 'Space-time discontinuous Galerkin finite element method with dynamic grid motion for inviscid compressible flows I. General formulation'. J. Comp. Phys. 182, 546-585. 


\section{A Summary of Exact Solutions}

In the following, we provide all the known exact solutions used in the main text for reference.

Consider a dam break problem with as initial condition $h\left(x,-t_{0}\right)=H_{0}$ for $x-x_{0}<$ 0 and zero elsewhere, and $u\left(x,-t_{0}\right)=0$. The terms on the RHS of (1.1) are zero, $S_{2}=S_{3}=0$. The evolving dam break solution in an unbounded domain is

$$
\begin{array}{r}
g h(x, t)=\left\{\begin{array}{lr}
a_{0}^{2} & x-x_{0}<-a_{0} t \\
\frac{1}{9}\left(2 a_{0}-\left(x-x_{0}\right) / t\right)^{2} & -a_{0}\left(t+t_{0}\right)<x-x_{0}<2 a_{0} t \\
0 & x-x_{0}>2 a_{0} t
\end{array}\right. \\
u(x, t)=\left\{\begin{array}{lr}
0 & x-x_{0}<-a_{0} t \\
\frac{2}{3}\left(a_{0}+\left(x-x_{0}\right) / t\right) & -a_{0} t<x-x_{0}<2 c_{0} t \\
0 & x-x_{0}>2 a_{0} t
\end{array}\right.
\end{array}
$$

with $a_{0}=\sqrt{g H_{0}}$.

The Riemann solution with $U=U_{l}$ for $x<x_{0}$ and $U=U_{r}$ for $x>x_{0}$ at $t=0$ in which a dry patch appears can be stated explicitly. The terms on the RHS of (1.1) are zero, $S_{2}=S_{3}=0$. Drying occurs when $c_{l}+c_{r}-u_{r}+u_{l}<0$, and the solution is:

$$
\begin{array}{r}
a(x, t)=\left\{\begin{array}{lr}
a_{l} & x-x_{0}<\left(u_{l}-a_{l}\right) t \\
\frac{1}{3}\left[u_{l}+c_{l}-\left(x-x_{0}\right) / t\right] & \left(u_{l}-a_{l}\right) t<x-x_{0}<S_{l} t \\
0 & S_{l} t<x-x_{0}<S_{r} t \\
\frac{1}{3}\left[\left(x-x_{0}\right) / t-u_{r}+c_{r}\right] & S_{r} t<x-x_{0}<\left(u_{r}+a_{r}\right) t \\
a_{r} & x-x_{0}>\left(u_{r}+a_{r}\right) t
\end{array}\right. \\
u(x, t)=\left\{\begin{array}{lr}
u_{l} & x-x_{0}<\left(u_{l}-a_{l}\right) t \\
\frac{1}{3}\left[u_{l}+c_{l}+2\left(x-x_{0}\right) / t\right] & \left(u_{l}-a_{l}\right) t<x-x_{0}<S_{l} t \\
0 & S_{l} t<x-x_{0}<S_{r} t \\
\frac{1}{3}\left[2\left(x-x_{0}\right) / t+u_{r}-c_{r}\right] & S_{r} t<x-x_{0}<\left(u_{r}+a_{r}\right) t \\
u_{r} & x-x_{0}>\left(u_{r}+a_{r}\right) t
\end{array}\right.
\end{array}
$$

with $a=\sqrt{g h}, c=2 a$ and $S_{l}=u_{l}+c_{l}, S_{r}=u_{r}-c_{r}$.

Consider a fluid in a parabolic bowl, which resides symmetrically around the origin. The following, scaled shallow water equations $\partial_{t} u+u \partial_{x} u=-\partial_{x}\left(h+h_{b}\right)$ and $\partial_{t} h+\partial_{x}(h u)=0$ can be simplified exactly by introducing the following Ansatz (Young, 1986): $u=u_{0}(t) x, h=h_{0}(t)-h_{1}(t) x^{2} / 2$, and $h_{b}=B x^{2} / 2$ with $x \in\left[-x_{b}, x_{b}\right]$ and, hence, $x_{b}(t)=\sqrt{2 h_{0}(t) / h_{1}(t)}$. Mass $M=\int h(x, t) \mathrm{d} x=(2 / 3) h_{1} x_{b}^{3}=(2 / 3) m$ is conserved, that is $d M / d t=0$. Given this conserved mass and an initial condition defining $m$, the above system can be reduced to:

$$
\frac{d x_{b}}{d t}=\frac{1}{2 x_{b}} \frac{\partial E}{\partial u_{0}}=u_{0} x_{b} \quad \frac{d u_{0}}{d t}=-\frac{1}{2 x_{b}} \frac{\partial E}{\partial x_{b}}=-u_{0}^{2}-B+m / x_{b}^{3}
$$

with "energy" $E=u_{0}^{2} x_{b}^{2}+B x_{b}^{2}+2 m / x_{b}$. Instead of finding a closed-from solution, we numerically integrate the two ordinary differential equations (A.5) directly for comparison with the solution of the finite-element discretization. 
Carrier and Greenspan (1958) considered the dimensionless shallow-water equations on a uniform slope $\partial_{t} h+\partial_{x}(h u)=0$ and $\partial_{t} u+u \partial_{x} u+\partial_{x} h=-1$. They introduced a hodograph transformation with $u=\lambda / 2-t$ and $c=\sqrt{h}=\tilde{\sigma} / 4$, such that the shallow-water equations reduce to a linear equation in $\phi$ with $u(\tilde{\sigma}, \lambda)=\partial_{\tilde{\sigma}} \phi / \tilde{\sigma}$. Hence the shoreline lies at $\tilde{\sigma}=0$. The solution is well defined provided the Jacobian between $x, t$ and $\tilde{\sigma}, \lambda$ exists, where

$$
t=\lambda / 2-u, \quad \text { and } \quad x=\partial_{\lambda} \phi / 4-\tilde{\sigma}^{2} / 16-u^{2} / 2 .
$$

Assuming $\phi$ to be harmonic in $\lambda$, the solution is a sum of Fourier modes:

$$
\phi(\tilde{\sigma}, \lambda)=\sum_{k=0}^{\infty} A_{k} J_{0}\left(\omega_{k} \tilde{\sigma}\right) \sin \left(\omega_{k} \lambda+\varphi_{k}\right)
$$

with $\omega_{k}$ the frequency and $J_{0}$ the Bessel function of the first kind and $\varphi_{k}$ a phase. We consider the case where the Jacobian of the transformation is critical for a single mode such that $A_{k}=1, \omega_{k}=1$ and the front reaches infinite steepness at periodic instants.

Peregrine and Williams (2001) considered the following solution overtopping a dike for the scaled shallow-water equations $\partial_{t} h+\partial_{x}(h u)=0$ and $\partial_{t} u+u \partial_{x} u+\partial_{x} h=-\partial_{x} h_{b}$ with $h_{b}(x)=1$ for $x<x_{0}+E$ and $h_{b}(x)=-1$ for $x \geq x_{0}+E$. Consider initially quiescent flow with depth unity for $x<x_{0}$ and no fluid or zero depth beyond. The critical point lies at $x_{0}+E$ with $0<E<1$. The following analytical solution emerges for $x<x_{0}+E$. For $t \in\left[0, T_{1}\right]$, where $T_{1}=2-\sqrt{4-2 E}$ the time when the front reaches the critical point at $x_{0}+E$, and for $t \in\left[T_{1}, T_{2}\right]$, where $T_{2}=\sqrt{2 E}$ the time when the flow becomes critical, one obtains:

$$
\begin{aligned}
& h(x, t)=\left\{\begin{array}{lr}
1 & x-x_{0}<-t^{2} / 2-t \\
\frac{1}{36 t^{2}}\left(4 t-t^{2}-2\left(x-x_{0}\right)\right)^{2} & -t^{2} / 2-t<x-x_{0}< \\
0 & \min \left(x_{s}-x_{0} \equiv-t^{2} / 2+2 t, E\right)
\end{array}\right. \\
& u(x, t)=\left\{\begin{array}{lr}
-t & \min \left(-t^{2} / 2+2 t, E\right)<x-x_{0}<E \\
\frac{2}{3 t}\left(t-t^{2}+\left(x-x_{0}\right)\right) & -t^{2} / 2-t<x-x_{0}<\min \left(-t^{2} / 2+2 t, E\right) . \\
0 & \min \left(-t^{2} / 2+2 t, E\right)<x-x_{0}<E
\end{array}\right.
\end{aligned}
$$

For $t \in\left[T_{2}, T_{3}\right]$ with $T_{3}=2$ the time when the flow starts to recede down the slope 
from the critical point, and for $t>T_{3}$, we find the exact solution:

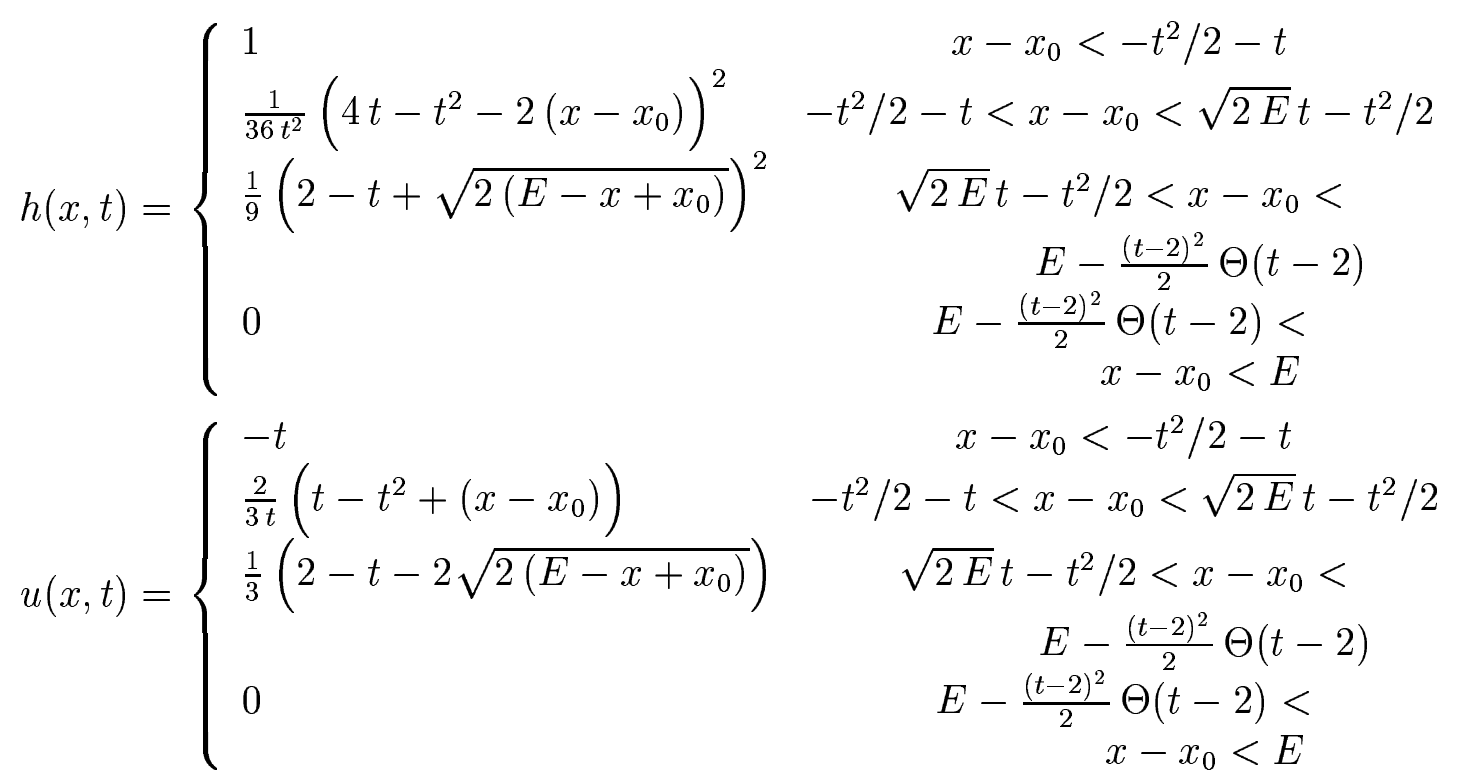

with Heaviside function $\Theta(\cdot)$.

\section{B Extra numerical dissipation}

The slope limiting treatment of Schwanenberg (2003) is akin to well-known slope limiters, but is applied to a formulation in terms of the variables $z_{s}=h+h_{b}, u, v$ instead of $h, u, v$ provided we choose the reference level such that $z_{s}(x, t)>0$. In terms of $z_{s}$ the shallow-water equations read

$$
\partial_{t} z_{s}+\partial_{x}\left(u\left(z_{s}-h_{b}\right)\right)=0, \quad \partial_{t} u+u \partial_{x} u+g \partial_{x} z_{s}=0
$$

Instead of the usual Riemann invariants $u \pm 2 \sqrt{g h}, u$ for the flat bottom shallow water equations, we use the approximations $u \pm 2 \sqrt{g z_{s}}, u$ to ensure that the solution is limited to a horizontal water surface and not a surface parallel to the topography. The limiter in the approximate characteristic variables $U_{c}$ limits the existing slope to the new slope $\hat{U}_{c}^{\prime}$

$$
\hat{U}_{c, k}^{\prime}=m\left(\hat{U}_{c, k}, \bar{U}_{c, k+1}-\bar{U}_{c, k}, \bar{U}_{c, k}-\bar{U}_{c, k-1}\right)
$$

with the minmod function $m(\cdot)$

$$
m\left(a_{1}, a_{2}, a_{3}\right)=\left\{\begin{array}{cc}
s \min _{1 \leq n \leq 3}\left|a_{n}\right| & \text { if } \\
0 & s=\operatorname{sign}\left(a_{1}\right)=\cdots=\operatorname{sign}\left(a_{n}\right) \\
\text { otherwise }
\end{array}\right.
$$

This limiter does performs but poorly with our flooding and drying scheme since it introduces extra uncontrollable dissipation in shallow areas in which shocks should have minimal or zero strength. Consequently, the accuracy decreases. Indeed, Shen 
and Meyer (1963) showed that the jump of a bore approaching a beach reduces to zero in the limit $h \rightarrow 0$. From the one-dimensional shock relation it follows that the shock speed becomes infinite when there is no water in front of the shock. Hence, the shock strength diminishes quickly when $h \rightarrow 0$.

Krivodonova et al. (2003) derive a shock detection criterion to selectively use extra stabilizing dissipation at shocks only. Their approach uses the difference in convergence rates between smooth and discontinuous parts of the solution. The following quantity is used for shock detection

$$
\mathcal{I}_{k}=\frac{\left|\left(\bar{h}_{k}-\hat{h}_{k}-\bar{h}_{k-1}-\hat{h}_{k-1}\right) \Theta\left(u_{k, L}, 0\right)+\left(\bar{h}_{k}+\hat{h}_{k}-\bar{h}_{k+1}+\hat{h}_{k+1}\right) \Theta\left(-u_{k, R}, 0\right)\right|}{\left|K_{k}\right||| h_{k} \|_{\max }}
$$

for the depth $h$, written in our one-dimensional notation with first-order polynomial basis functions. Here $u_{k, L}$ is the velocity in element $K_{k}$ at the left face at $x_{k}$ and $u_{k, R}$ the velocity in $K_{k}$ at the right face at $x_{k+1}$. $\|h\|_{\text {max }}$ denotes the maximum of $h$ in element $K_{k}$. The quantity $\mathcal{I}_{k}$ signals whether the (numerical) jumps of $h$ that move into an element $K_{k}$ correspond to genuine discontinuities or not. The detection scheme of Krivodonova et al. (2003) states that:

$$
h=\left\{\begin{array}{ccc}
\text { is discontinuous } & \text { if } & \mathcal{I}_{k}>1 \\
\text { is continuous } & \text { if } & \mathcal{I}_{k}<1
\end{array}\right.
$$

only when $\mathcal{I}_{k}>1$ the previous limiter is activated. We have used the depth as tell-tale. Other choices are used by Krivodonova et al. (2003) in the context of the compressible Euler equations. 


\begin{tabular}{c|c|c|c|c|c|c|c|c|c}
$N$ & $L^{2}(u, h)$ & $p$ & $L^{2}(m, h)$ & $p$ & $L^{\infty}$ & $p$ & $\epsilon_{x_{R}}$ & $p$ \\
\hline 50 & 0.030743 & & 0.019413 & & 0.09060 & & 1.9698 & \\
100 & 0.019316 & 0.67 & 0.010832 & 0.84 & 0.08300 & 0.13 & 1.3194 & 0.58 \\
200 & 0.011859 & 0.70 & 0.005860 & 0.89 & 0.06351 & 0.39 & 0.8747 & 0.59 \\
400 & 0.007155 & 0.73 & 0.003106 & 0.92 & 0.04631 & 0.46 & 0.5570 & 0.65 \\
800 & 0.004200 & 0.77 & 0.001619 & 0.94 & 0.03438 & 0.43 & 0.3391 & 0.72
\end{tabular}

Table 1: Dam break test without extra dissipation. Error in norms $L^{2}(u, h), L^{2}(m, h)$, $L^{\infty}$; and the frontal position, $\epsilon_{x_{R}}=x_{R}-x_{R}^{\text {numerical }}$, at time $t=4$. Parameters and initial conditions are $x<x_{0}: u(x, 0)=0, h(x, 0)=1 ; x>x_{0}: u(x, 0)=0, h(x, 0)=0$ with $x_{0}=10, a_{0}=H_{0}=1, C F L=0.1$. 


\begin{tabular}{c|c|c|c|c|c}
$N$ & $L^{2}(u, h)$ & $p$ & $L^{2}(m, h)$ & $p$ & $L^{\infty}$ \\
\hline 50 & 0.2423 & & 0.074455 & & 1.630 \\
100 & 0.1558 & 0.64 & 0.026786 & 1.47 & 1.549 \\
200 & 0.1831 & -0.23 & 0.014764 & 0.86 & 1.523 \\
400 & 0.1475 & 0.31 & 0.007556 & 0.97 & 1.499 \\
800 & 0.1550 & -0.07 & 0.004023 & 0.91 & 1.496
\end{tabular}

Table 2: Drying test without extra dissipation. Error in norms $L^{2}(u, h), L^{2}(m, h)$, $L^{\infty}$; and the frontal position closest to the origin, $\epsilon_{x_{R}}=x_{R}-x_{R}^{\text {numerical }}$, at time $t=1.5$. Parameters and initial conditions $x<x_{0}: u(x, 0)=u_{l}, h(x, 0)=h_{l} ; x>x_{0}$ : $u(x, 0)=u_{r}, h(x, 0)=h_{r}$ with $h_{l}=0.5, h_{r}=1, u_{l}=0, u_{r}=3.5, c_{0}=1, C F L=0.5$. The drying criterion $c_{l}+c_{r}-u_{r}+u_{l} \approx-8.5786 \mathrm{e}-2<0$ is met. 


\begin{tabular}{c|c|c|c|c|c|c|c}
$N$ & $L^{2}(u, h)$ & $p$ & $L^{2}(m, h)$ & $p$ & $L^{\infty}$ & $p$ & $\epsilon_{x_{b}}$ \\
\hline & & & & & \\
10 & 0.03891532 & & 0.03637109 & & 0.0678214 & & -0.01737615 \\
20 & 0.01450206 & 1.42 & 0.00653306 & 2.48 & 0.0338388 & 1.00 & -0.00276955 \\
40 & 0.00229621 & 2.66 & 0.00129805 & 2.33 & 0.0053047 & 2.67 & -0.00083484 \\
80 & 0.00058647 & 1.97 & 0.00033048 & 1.97 & 0.0019417 & 1.45 & -0.00007639 \\
160 & 0.00014010 & 2.07 & 0.00010330 & 1.68 & 0.0005360 & 1.86 & -0.00010299 \\
320 & 0.00002367 & 2.57 & 0.00001827 & 2.50 & 0.0001101 & 2.28 & -0.00001834
\end{tabular}

Table 3: Parabolic bowl test without extra dissipation. Error in the norms $L^{2}(u, h)$, $L^{2}(m, h), L^{\infty}$; and the error in the frontal position $x_{b}, \epsilon_{x_{b}}=x_{b}-x_{b}^{\text {numerical }}$, all at time $t=3$. Parameters and initial conditions are $u(x, 0)=0, h(x, 0)=0.5 h_{1}(0)\left(x_{b}(0)-\right.$ $\left.(x-0.5)^{2}\right)$ with $B=12, x_{b}(0)=\sqrt{1 / 6}, h_{1}(0)=12, C F L=0.01$. 


\begin{tabular}{c|c|c|c|c|c|c|c|c|c}
$N$ & $L^{2}(u, h)$ & $p$ & $L^{2}(m, h)$ & $p$ & $L^{\infty}$ & $p$ & $\epsilon_{x_{R}}$ & $p$ \\
\hline & & & & & & \\
10 & 0.00373049 & & 0.00178246 & & 0.0071550 & & -0.0055263 & \\
20 & 0.00090234 & 2.05 & 0.00047815 & 1.90 & 0.0019300 & 1.89 & -0.0019313 & 1.51 \\
40 & 0.00052466 & 0.78 & 0.00041581 & 0.20 & 0.0014153 & 0.45 & -0.0007257 & 1.41 \\
80 & 0.00025871 & 1.02 & 0.00022217 & 0.90 & 0.0007413 & 0.93 & -0.0003197 & 1.18 \\
160 & 0.00005417 & 2.26 & 0.00003680 & 2.59 & 0.0003948 & 0.91 & -0.0001476 & 1.11 \\
320 & 0.00002251 & 1.27 & 0.00001020 & 1.85 & 0.0002998 & 0.40 & -0.0000611 & 1.27 \\
640 & 0.00001180 & 0.93 & 0.00000637 & 0.68 & 0.0002178 & 0.46 & -0.0000258 & 1.24
\end{tabular}

Table 4: Carrier-Greenspan test without extra dissipation. Error in norms $L^{2}(u, h)$, $L^{2}(m, h), L^{\infty}$; and the error on the frontal position $x_{R}, \epsilon_{x_{R}}=x_{R}-x_{R}^{\text {numerical }}$, all at time $t=4$. $C F L=0.01$. For the other parameters and initial conditions see Appendix A. 


\begin{tabular}{c|c|c|c|c|c|c|c|c|c|c}
$\frac{N}{10}$ & $\frac{L^{2}(u, h)}{10^{-2}}$ & $p$ & $\frac{L^{2}(m, h)}{10^{-2}}$ & $p$ & $\frac{L^{\infty}}{10^{-2}}$ & $p$ & $\epsilon_{x_{s}}$ & $p$ & $\frac{\Delta t_{b}}{10^{-2}}$ & $p$ \\
\hline 5 & 1.11062 & & 0.27475 & & 3.5526 & & 0.11229 & & 1.8016 & \\
10 & 0.25262 & 2.13 & 0.01023 & 1.43 & 0.8717 & 2.03 & 0.09737 & 0.21 & 2.2975 & -0.3508 \\
20 & 0.12717 & 0.99 & 0.04828 & 1.08 & 0.6081 & 0.52 & 0.06290 & 0.63 & 1.9403 & 0.24 \\
40 & 0.06738 & 0.91 & 0.02356 & 1.04 & 0.4991 & 0.29 & 0.03995 & 0.65 & 1.3653 & 0.51 \\
80 & 0.03680 & 0.87 & 0.01160 & 1.02 & 0.3877 & 0.36 & 0.02502 & 0.68 & 0.9631 & 0.50
\end{tabular}

Table 5: The Peregrine-Williams overtopping test without additional dissipation. Error in norms $L^{2}(u, h), L^{2}(m, h), L^{\infty}$; and the frontal position of the first patch, $\epsilon_{x_{s}}=x_{s}-x_{s}^{\text {numerical }}$ with $x_{s}=x_{0}-t^{2} / 2+2 t$, at time $t=3$, and the time of break up, $\Delta t_{b}$. The exact solution breaks at $t=2$. Parameters and initial conditions $L=$ $4 ; x_{0}=2 ; E=1 ; x<x_{0}: u(x, 0)=-1, h(x, 0)=1 ; x>x_{0}: u(x, 0)=0, h(x, 0)=0$ and $C F L=0.01$. 


\section{Figure Captions}

Figure 1

The wave configuration is sketched to define the variables and states involved in the HLLC flux approximation. The constant left and right states $U_{l, r}$ are separated by a left and right wave $S_{l, r}$ from two star regions $U_{l, r}^{*}$. These two star regions are divided by a contact wave $S_{m}$.

Figure 2

A sketch of the $M$ patches $P_{i}$ within the space $\Omega$.

Figure 3

A patch in the finite-element space with a free-boundary element $K_{\mathrm{ri}}$ on the right. The (interior) elements away from a free boundary are fixed or Eulerian, while the free-boundary element is mixed Eulerian-Lagrangian. Hence, the computational mesh used is a particular case of an ALE mesh (e.g., Van der Vegt and Van der Ven, 2002). Denoted is the height field $h(x, t)$ for the case with linear basis functions. Note that in $K_{\text {ri }}$ we have $h=0$ at the free boundary.

Figure 4

Element a) splitting and b) merging sketched for the depth field in the case of linear basis functions.

Figure 5

Patch splitting sketched for the depth field in the case of linear basis functions.

Figure 6

Patch merging sketched for the depth field for two generic cases where free boundaries overlap a) in one element and b) in two elements in the case of linear basis functions.

\section{Figure 7}

Free surface and velocity profiles are shown as functions of space and time for the dam break problem. Solid lines display the numerical solution, and dashed lines the exact solution. 400 elements are used without additional numerical dissipation.

Figure 8

Free surface and velocity profiles are shown as functions of space and time for the Riemann problem in which a dry patch emerges. Solid lines display the numerical solution, and dashed lines the exact solution. 400 elements are used without additional numerical dissipation.

Figure 9

Free surface (40 elements) and velocity (20 and 40 elements) profiles are shown as functions of space and time for the parabolic bowl problem. Solid lines display the numerical solution, and thin and thick dashed lines the exact solution and the beach topography, respectively. No additional numerical dissipation is added. 


\section{Figure 10}

Free surface and velocity profiles for the Carrier-Greenspan test case, see Table 4, for $N=40$ elements without additional dissipation.

\section{Figure 11}

Free surface and velocity profiles are shown as functions of space and time for the Peregrine-Williams solution. $N=200$ elements are used with the HLLC flux without additional numerical dissipation. The (scaled) beach topography has slope $1: 1$, while this slope should be small in dimensional terms.

Figure 12

Two free surface profiles (thick solid lines) are shown from the front and back side of a dike, as well as velocity profiles for multiple waves steepening, running-up a dike and overtopping a dike. 400 elements are used with a localized slope limiter (combining Schwanenberg, 2003, and Krivodonova et al., 2003, see Appendix B). Slope limiting is not necessary for the robustness of the method, but avoids the modest oscillations around the incoming bores occurring otherwise. Note how periodic the space-time profiles become. The (scaled) beach topography is indicated by the dashed lines. We observe that the patches of water are rushing down the back slope very fast and are very thin. 


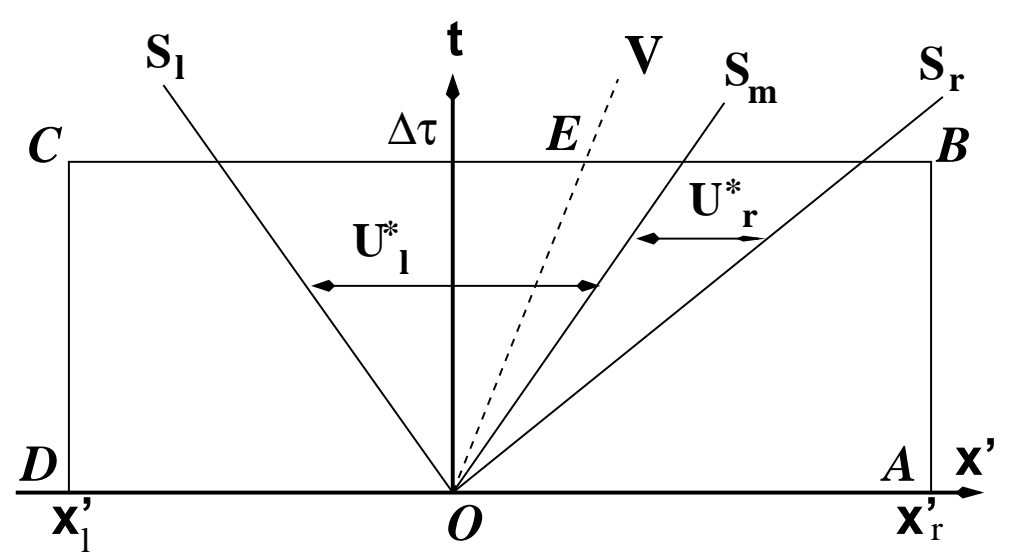

Figure 1: 


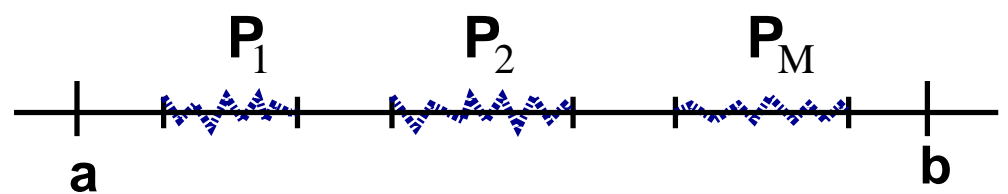

Figure 2: 


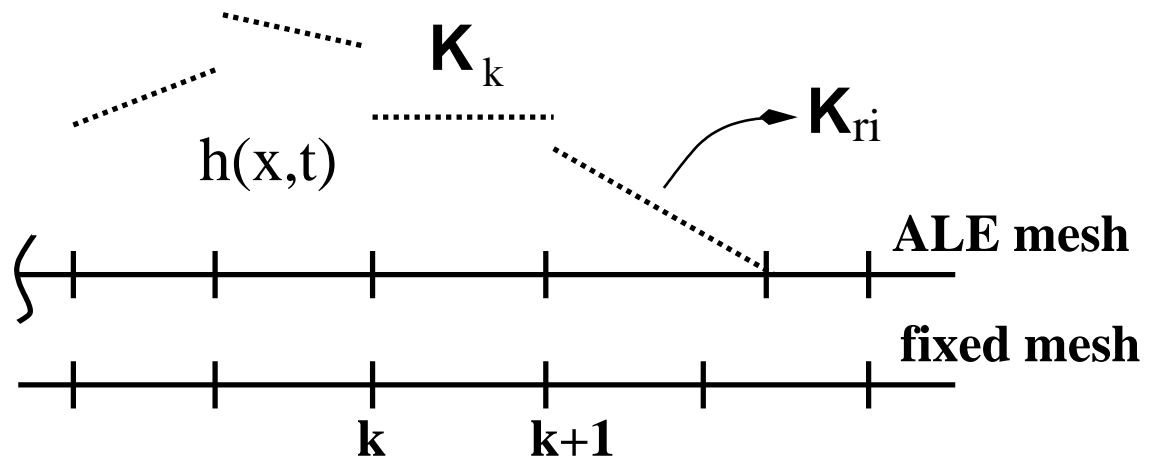

Figure 3: 
a)

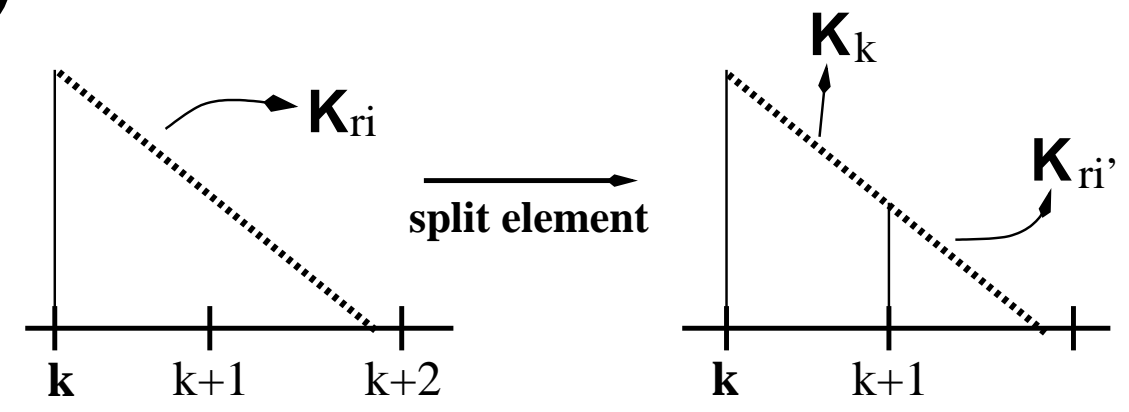

b)

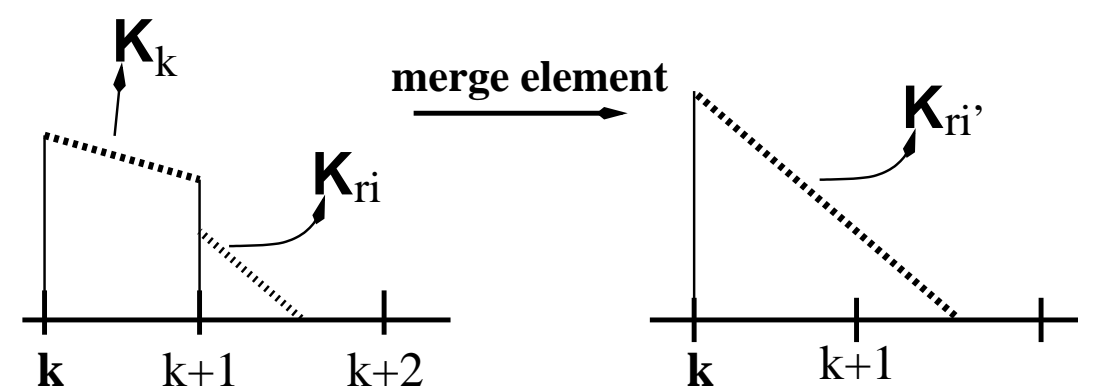

Figure 4: 

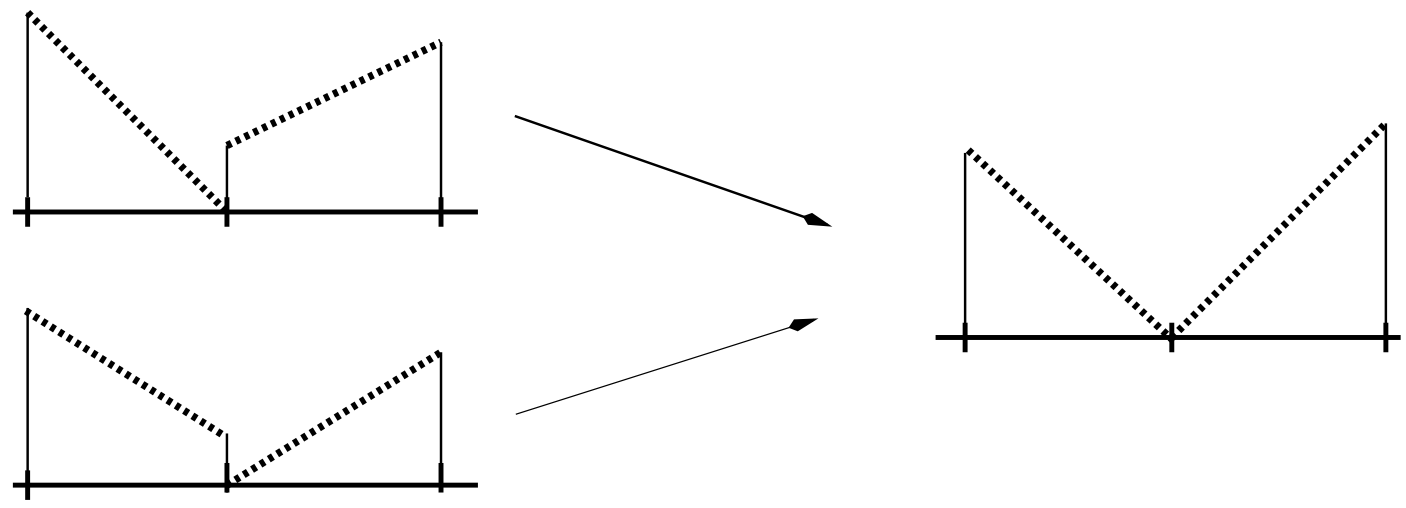

Figure 5: 
a)

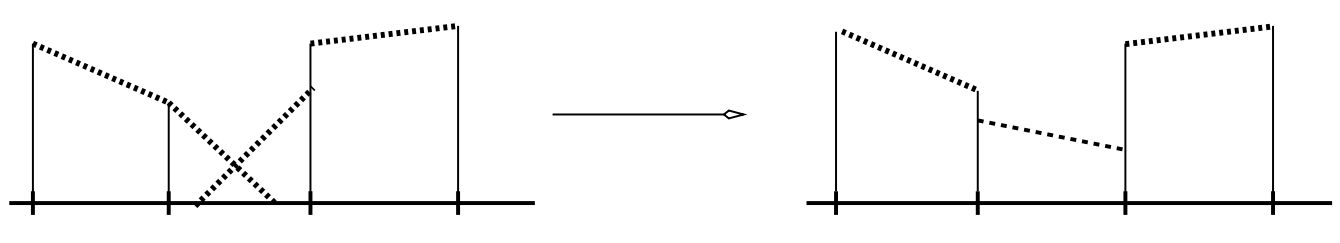

b)

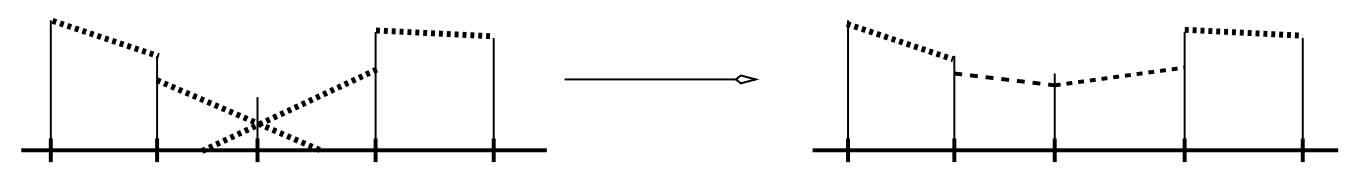

Figure 6: 


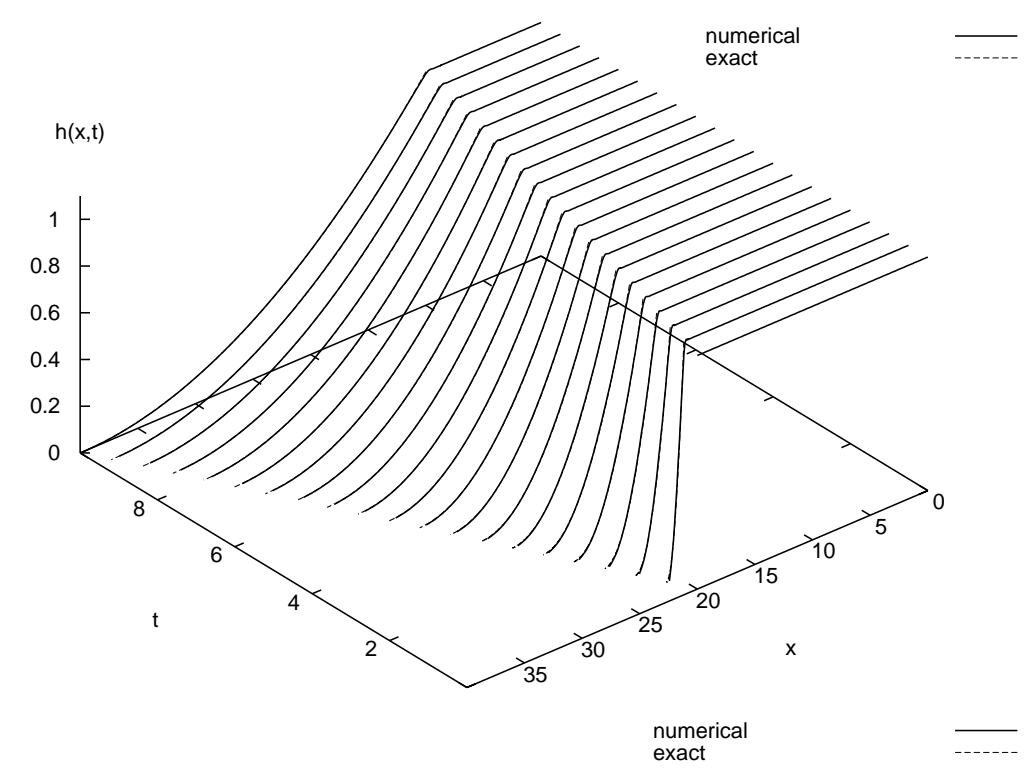

$u(x, t)$

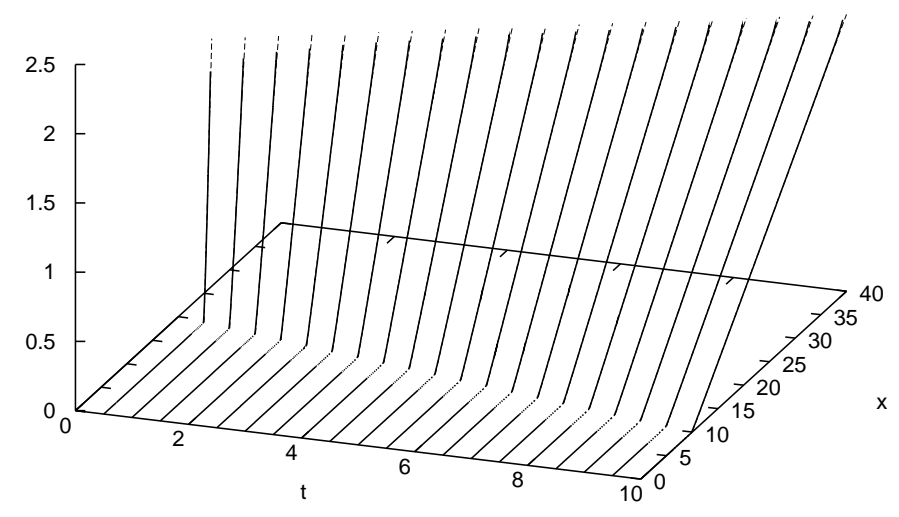

Figure 7: 

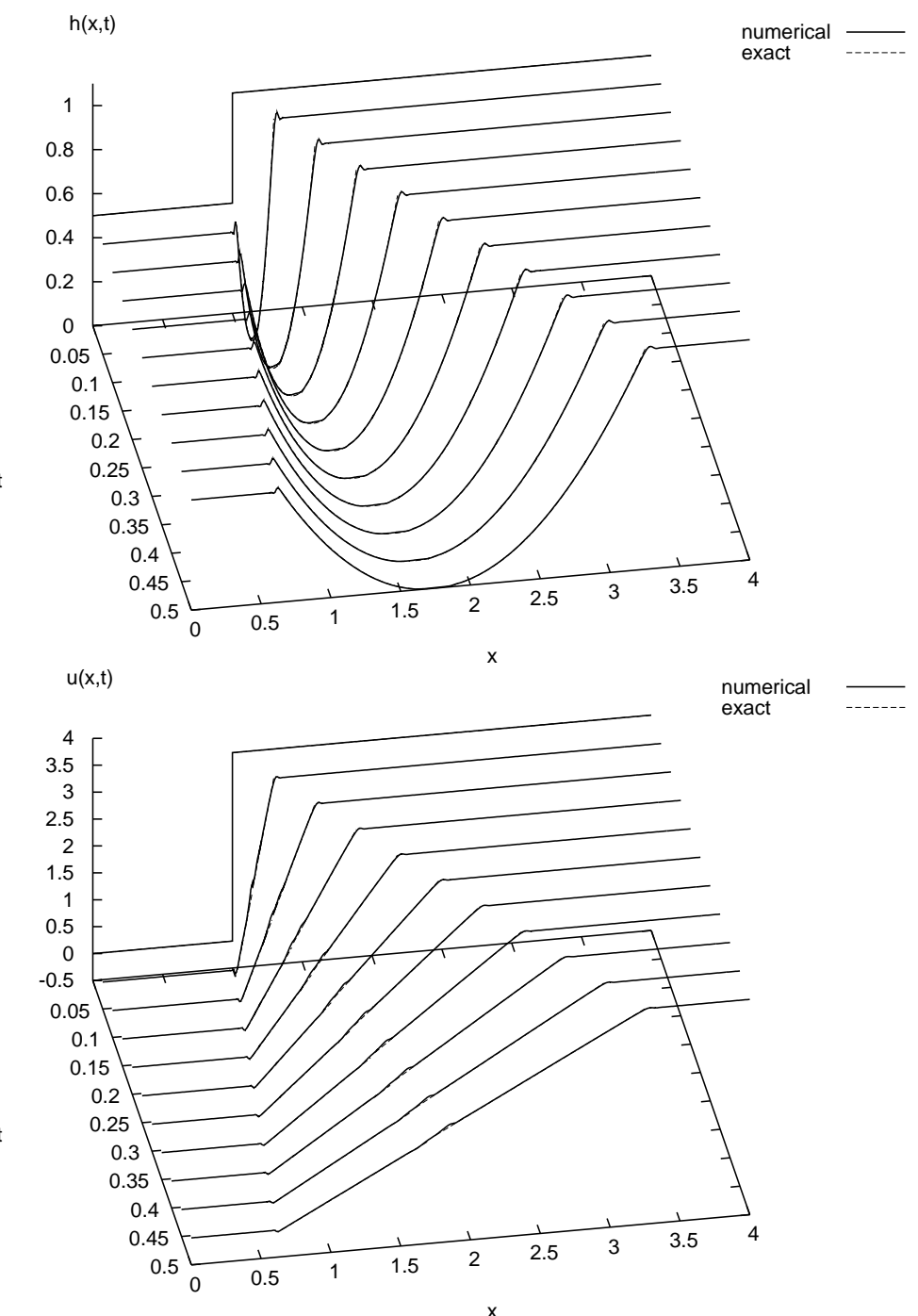

Figure 8: 

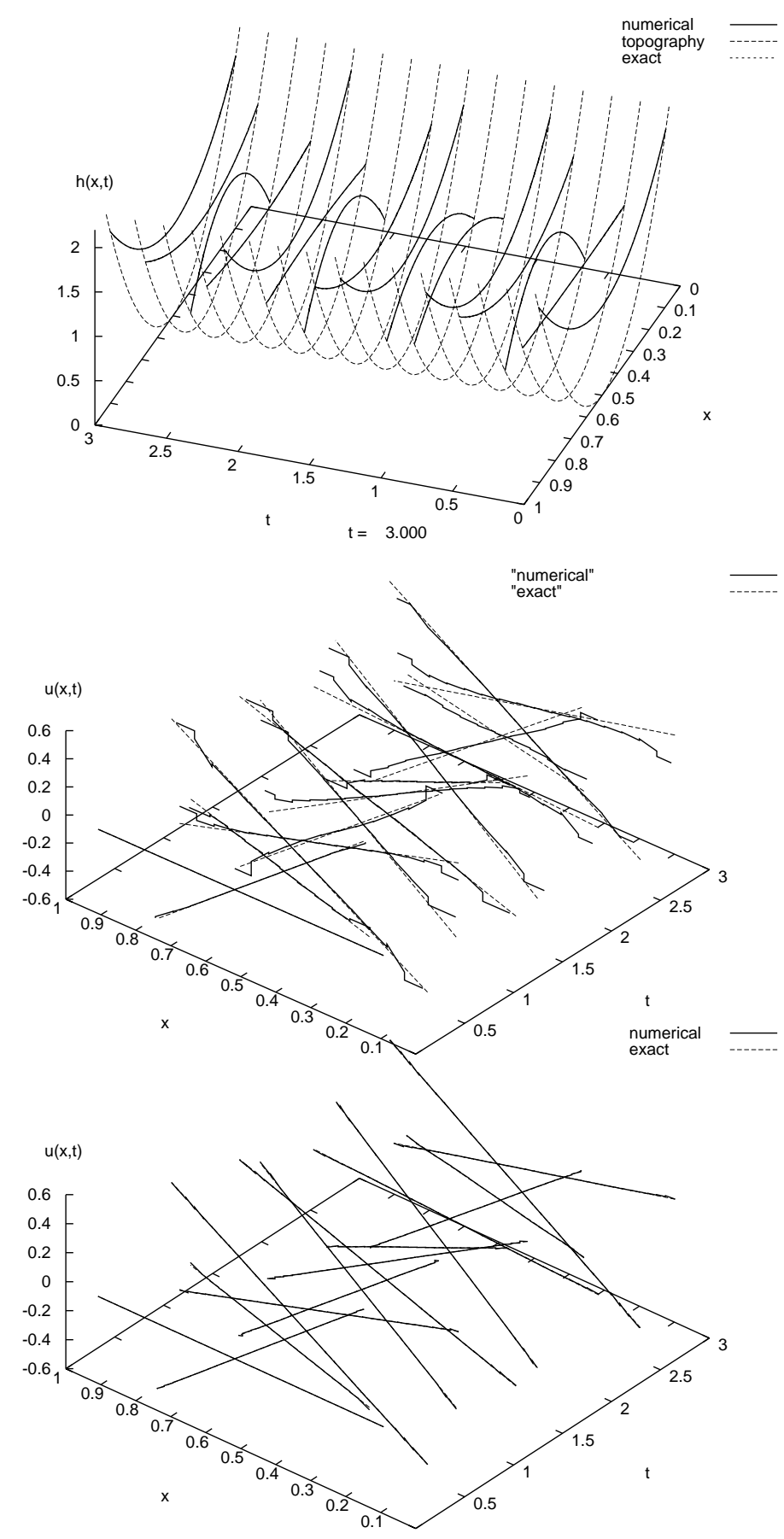

Figure 9: 

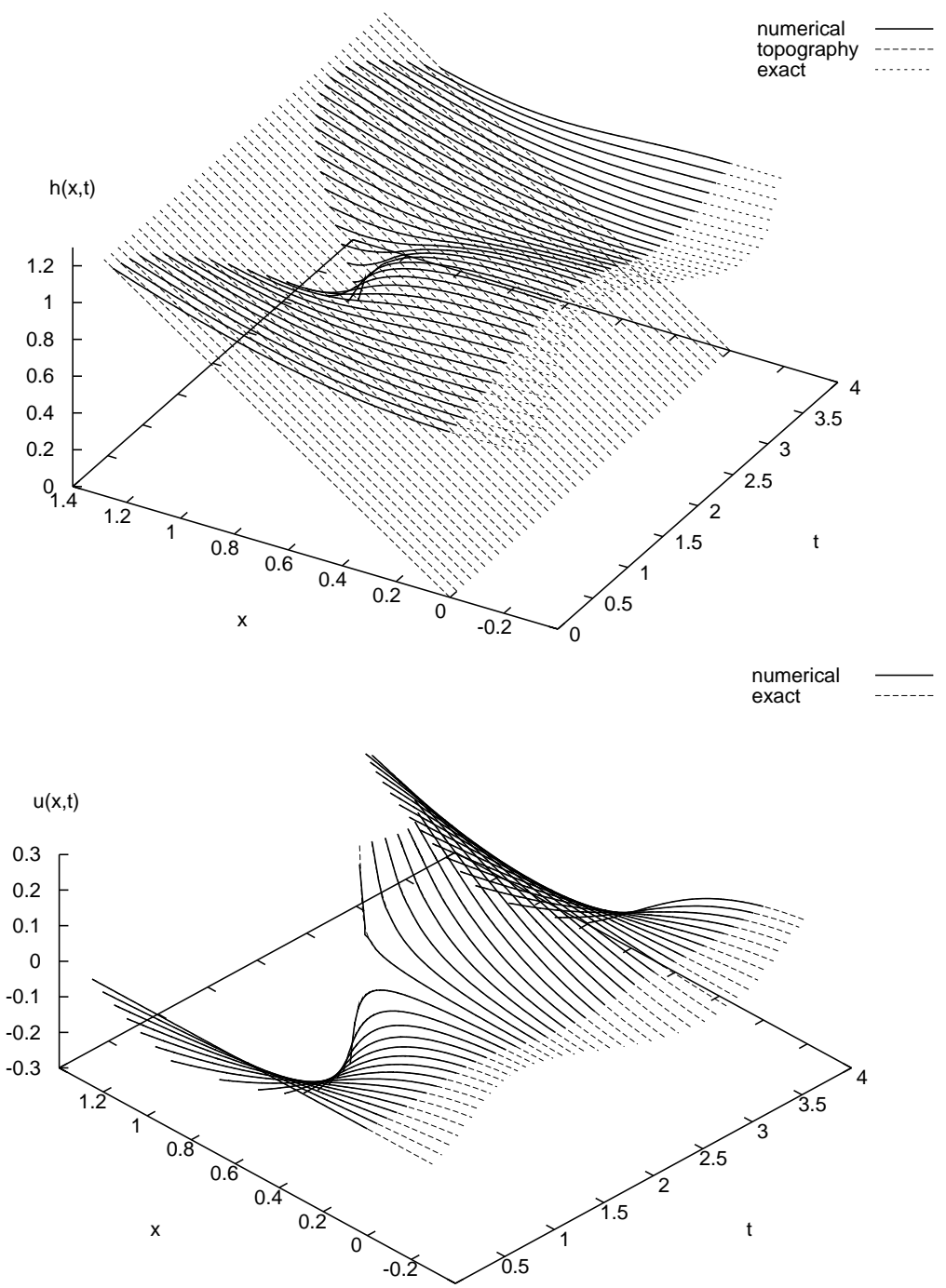

Figure 10: 

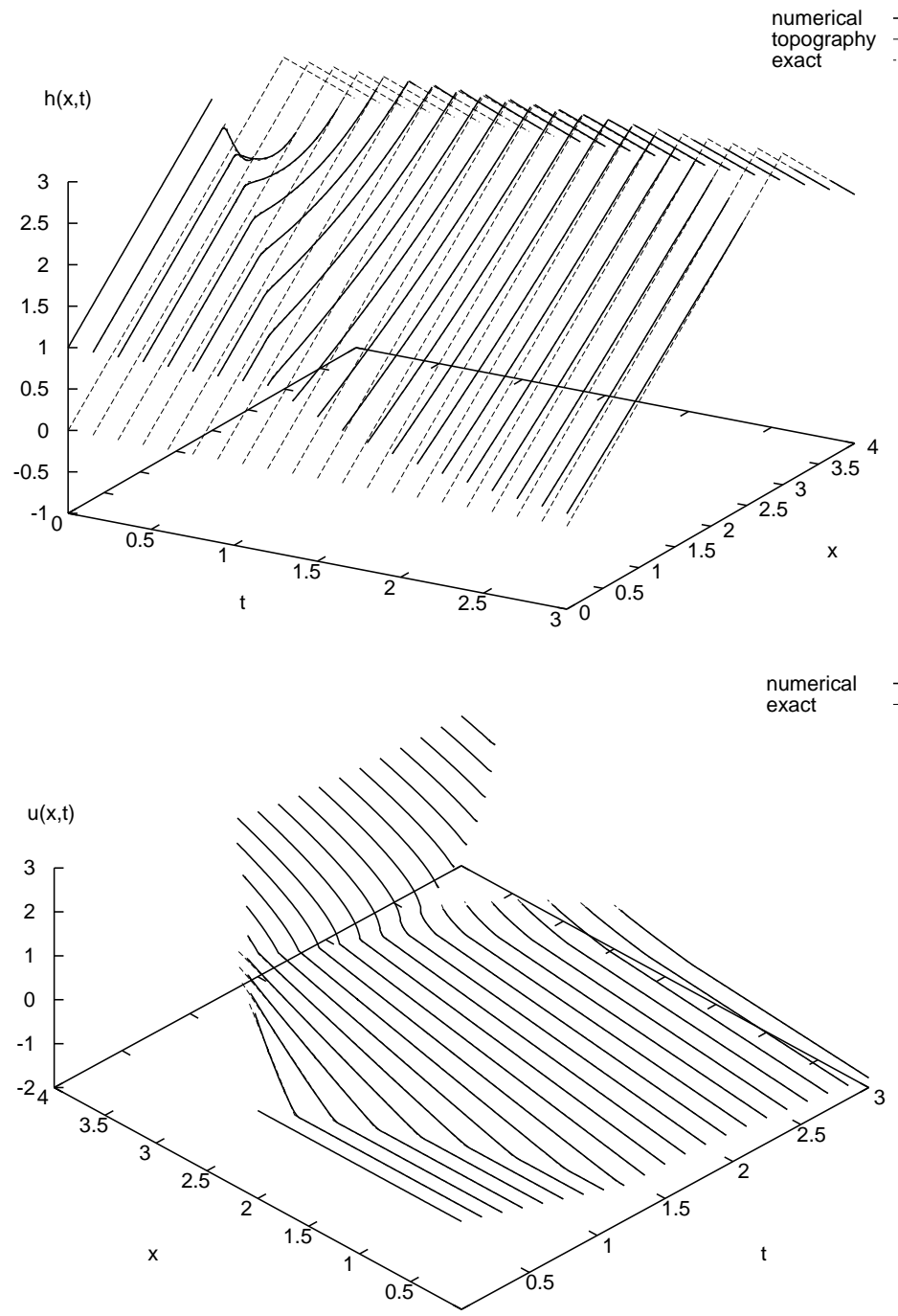

Figure 11: 

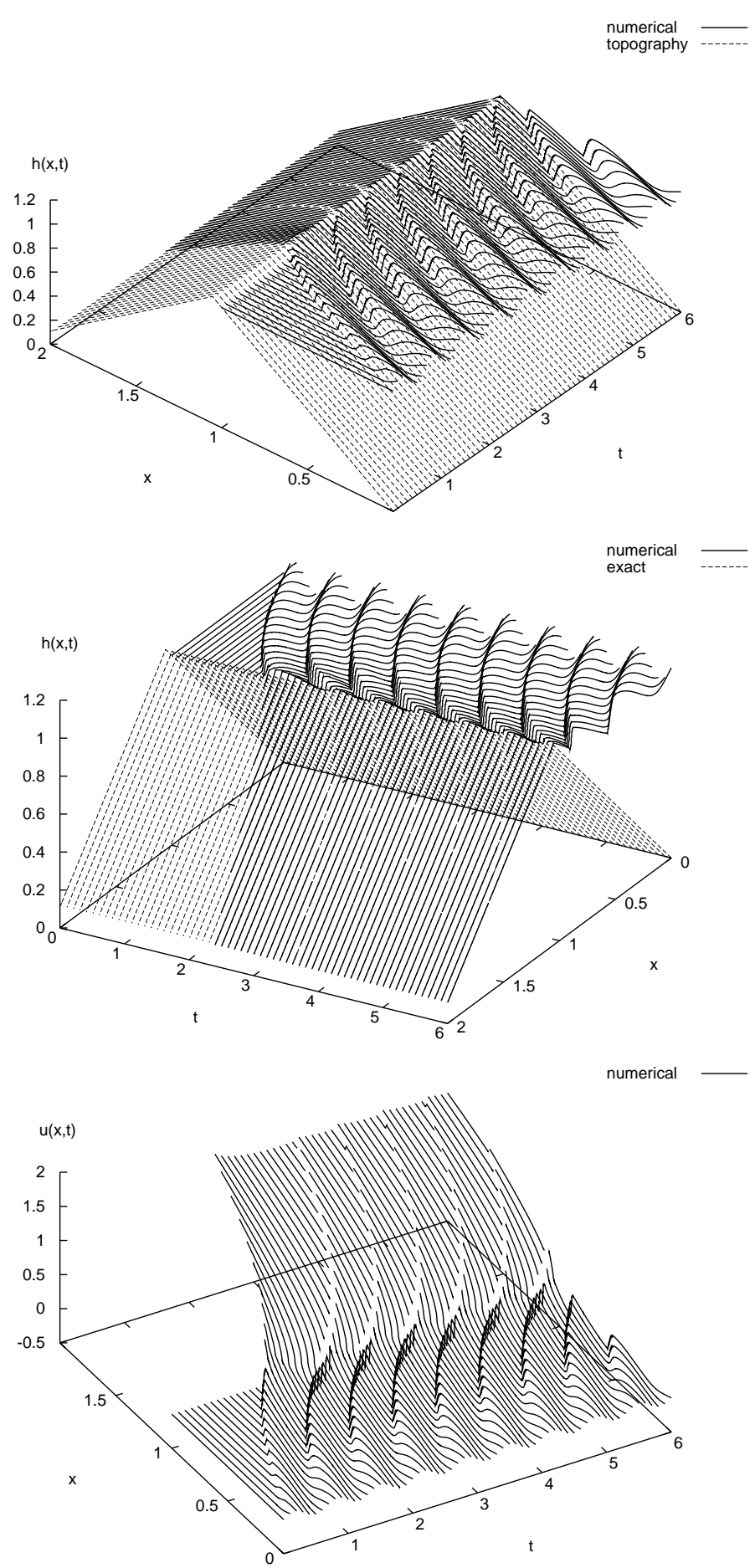

Figure 12: 